TRANSACTIONS OF THE

AMERICAN MATHEMATICAL SOCIETY

Volume 350, Number 12, December 1998, Pages 4869-4896

S $0002-9947(98) 02348-4$

\title{
HOMOLOGY OF THE CONFIGURATION SPACES OF QUASI-EQUILATERAL POLYGON LINKAGES
}

\author{
YASUHIKO KAMIYAMA, MICHISHIGE TEZUKA, AND TSUGUYOSHI TOMA
}

\begin{abstract}
We consider the configuration space $M_{n, r}$ of quasi-equilateral polygon linkages with $n$ vertices each edge having length 1 except for one fixed edge having length $r(r \geq 0)$ in the Euclidean plane $\mathbf{R}^{2}$. In this paper, we determine $H_{*}\left(M_{n, r} ; \mathbf{Z}\right)$.
\end{abstract}

\section{INTRODUCTION}

We consider the configuration space $M_{n, r}$ of quasi-equilateral polygon linkages with $n$ vertices, each edge having length 1 except for one fixed edge having length $r(r \geq 0)$ in the Euclidean plane $\mathbf{R}^{2}$. More precisely, we define $\mathcal{C}_{n, r}(n \geq 1)$ by

$$
\begin{aligned}
\mathcal{C}_{n, r}=\left\{\left(u_{1}, \ldots, u_{n}\right) \in\left(\mathbf{R}^{2}\right)^{n}:\right. & \left|u_{i+1}-u_{i}\right|=1 \quad(2 \leq i \leq n-1), \\
& \left.\left|u_{1}-u_{n}\right|=1, \text { and }\left|u_{2}-u_{1}\right|=r\right\} .
\end{aligned}
$$

Note that $\mathrm{Iso}^{+}\left(\mathbf{R}^{2}\right)$ (= the orientation preserving isometry group of $\mathbf{R}^{2}$, i.e., a semidirect product of $\mathbf{R}^{2}$ with $\left.S O(2)\right)$ naturally acts on $\mathcal{C}_{n, r}$. We define $M_{n, r}$ by

$$
M_{n, r}=\mathcal{C}_{n, r} / I_{s o}{ }^{+}\left(\mathbf{R}^{2}\right) \text { for } r>0 \text {, and } M_{n, 0}=\mathcal{C}_{n, 0} / \mathbf{R}^{2} .
$$

Then it is clear that $M_{n, r}(r \geq 0)$ can be described as follows:

$$
M_{n, r}=\left\{\left(u_{1}, \ldots, u_{n}\right) \in \mathcal{C}_{n, r}: u_{1}=\left(\frac{r}{2}, 0\right), u_{2}=\left(-\frac{r}{2}, 0\right)\right\} .
$$

As $M_{n, n-1}=\{1$-point $\}$, and $M_{n, r}=\emptyset(r>n-1)$, we can assume that $r<n-1$. Concerning $M_{n, 1}$, we have the following examples:

(i) $M_{3,1}=\{2$-points $\}$.

(ii) It is easy to see that $M_{4,1}$ is homeomorphic to $\left\{(x, y) \in \mathbf{R}^{2}:(x+1)^{2}+y^{2}=\right.$ 1\} $\cup\left\{(x, y) \in \mathbf{R}^{2}:(x-1)^{2}+y^{2}=1\right\} \cup\left\{(x, y) \in \mathbf{R}^{2}: x^{2}+y^{2}=4\right\}$.

(iii) It is well known that $M_{5,1}$ is diffeomorphic to $\Sigma_{4}$, i.e., the compact, connected, and orientable two dimensional manifold of genus 4 (see for example [2], [3], [4]).

The dimension and the smoothness of $M_{n, r}$ are studied in [5] (cf. Proposition 2.3 ), where the Euler characteristics of $M_{n, r}$ are also determined. Finally, $M_{5, r}$ is treated in [11].

The purpose of this paper is to determine the homology group of $M_{n, r}$. The results are as follows.

Received by the editors December 21, 1995.

1991 Mathematics Subject Classification. Primary 55R55; Secondary 51N20.

Key words and phrases. Polygon, linkage, homology. 


\section{Theorem A.}

(i) For $r \notin \mathbf{N}, M_{2 m+1, r}$ is homeomorphic to $M_{2 m+1, k_{1}}$, where $k_{1}$ is the odd number which satisfies $r-1<k_{1}<r+1$.

(ii) For $r \notin \mathbf{N}, M_{2 m, r}$ is homeomorphic to $M_{2 m, k_{2}}$, where $k_{2}$ is the even number which satisfies $r-1<k_{2}<r+1$.

By Theorem A, we need to know only $H_{*}\left(M_{n, k} ; \mathbf{Z}\right)$, where $n \geq 3$ and $k \in$ $\mathbf{N} \cup\{0\}$, in order to determine $H_{*}\left(M_{n, r} ; \mathbf{Z}\right)$. Concerning $H_{*}\left(M_{n, k} ; \mathbf{Z}\right)$, first we have the following:

Theorem B. $H_{*}\left(M_{n, k} ; \mathbf{Z}\right)$ is a torsion free module.

Thus in order to determine $H_{*}\left(M_{n, k} ; \mathbf{Z}\right)$, we need to describe the Poincaré polynomial $P S(n, k)$ of $M_{n, k}$, i.e., $P S(n, k)=\sum_{\lambda}$ rank $H_{\lambda}\left(M_{n, k} ; \mathbf{Z}\right) t^{\lambda}$. Actually they are determined in Theorem 6.8. In particular, we have the following result for $k=1$.

Theorem C. We have

$P S(2 m+1,1)=\sum_{\lambda=0}^{m-2}\left(\begin{array}{c}2 m \\ \lambda\end{array}\right) t^{\lambda}+2\left(\begin{array}{c}2 m \\ m-1\end{array}\right) t^{m-1}+\sum_{\lambda=m}^{2 m-2}\left(\begin{array}{c}2 m \\ \lambda+2\end{array}\right) t^{\lambda} \quad(m \geq 0)$

(ii) $P S(2 m, 1)=\sum_{\lambda=0}^{m-2}\left(\begin{array}{c}2 m-1 \\ \lambda\end{array}\right) t^{\lambda}+\left(\begin{array}{c}2 m \\ m-1\end{array}\right) t^{m-1}+\sum_{\lambda=m}^{2 m-3}\left(\begin{array}{c}2 m-1 \\ \lambda+2\end{array}\right) t^{\lambda} \quad(m \geq 1)$,

where $\left(\begin{array}{l}a \\ b\end{array}\right)$ is the binomial coefficient.

Theorem C suggests that the Lefschetz hyperplane section theorem and the (partial) Poincaré duality might hold for $M_{n, 1}$.

We recall the Lefschetz hyperplane section theorem (see [8]). Let $V$ be a smooth algebraic variety of complex dimension $l$ in $\mathbf{C} P^{N}$. Let $P$ be a hyperplane in $\mathbf{C} P^{N}$. Then the maps $H_{q}(V \cap P ; \mathbf{Z}) \rightarrow H_{q}(V ; \mathbf{Z})$ induced from the inclusion are isomorphisms for $q \leq l-2$ and an epimorphism for $q=l-1$.

By setting $z_{i}=u_{i+2}-u_{i+1}(1 \leq i \leq n-2), z_{n-1}=u_{1}-u_{n}$, and identifying $\mathbf{R}^{2}$ with $\mathbf{C}$, we can write $M_{n, 1}$ as

$$
M_{n, 1} \cong\left\{\left(z_{1}, \ldots, z_{n-1}\right) \in\left(S^{1}\right)^{n-1}: z_{1}+\cdots+z_{n-1}-1=0\right\} .
$$

If we regard $\left(S^{1}\right)^{n-1}$ as a "variety" and $\left\{\left(z_{1}, \ldots, z_{n-1}\right) \in(\mathbf{C})^{n-1}: z_{1}+\cdots+z_{n-1}-\right.$ $1=0\}$ as a "hyperplane", then Theorem C might be indicating some Lefschetz-type theorem for $M_{n, 1}$.

Concerning the (partial) Poincaré duality, as $M_{2 m+1,1}$ is a smooth manifold of dimension $2 m-2$ (cf. Proposition 2.3), Theorem $\mathrm{C}$ implies that $M_{2 m+1,1}$ is orientable, and hence satisfies the Poincaré duality. On the other hand, $M_{2 m, 1}$ is a manifold of dimension $2 m-3$ with isolated singular points. However, we have $H_{2 m-3}\left(M_{2 m, 1} ; \mathbf{Z}\right) \cong \mathbf{Z}$. If we choose the fundamental class $\left[M_{2 m, 1}\right]$, it seems that the Poincaré duality homomorphisms $\bigcap\left[M_{2 m, 1}\right]: H^{q}\left(M_{2 m, 1} ; \mathbf{Z}\right) \rightarrow H_{2 m-3-q}\left(M_{2 m, 1} ; \mathbf{Z}\right)$ are isomorphisms for $q \leq m-3$ or $q \geq m$. Such situation occurs if $M_{2 m, 1}$ is an orientable $(m-2)$-regular space in the sense of [7].

In fact, we can prove these facts in a different way. We will give their proofs in a subsequent paper. We note that we can prove Theorem $\mathrm{C}$ from the two theorems together with the Euler characteristics $\chi\left(M_{n, 1}\right)$. 


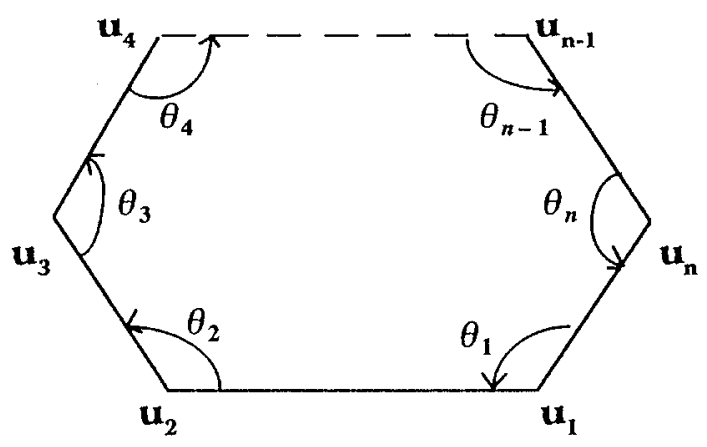

Figure 1

This paper is organized as follows. In $\S 2$, we define a map $\pi_{n, r}: M_{n, r} \rightarrow S^{1}$ for $r>0$, and study the singular fibers of it. Then we prove Theorem A. Next we prepare some notations which are used in later sections.

In $\S 3$, we construct deformations $f_{n, r}^{k}: M_{n, k} \rightarrow M_{n, r}$ for $n-k \equiv 0 \bmod 2$ and $r \in(k-1, k+1)$ with $k \in \mathbf{N} \cup\{0\}$. This $f_{n, r}^{k}$ has limits $\lim _{r \rightarrow k-1+0} f_{n, r}^{k}: M_{n, k} \rightarrow M_{n, k-1}$ and $\lim _{r \rightarrow k+1-0} f_{n, r}^{k}: M_{n, k} \rightarrow M_{n, k+1}$. We denote the former limit by $f_{n, k}^{-}$, and the latter limit by $f_{n, k}^{+}$. We prove this in Theorem 3.1.

In $\S 4$, we first define the homotopy colimit of a diagram. Theorem 3.1 asserts that $M_{n, k}$ is actually described in the language of homotopy colimit. We prove this in Theorem 4.6. At the same time, using this description, we prove in Theorem 4.7 that $f_{n, k}^{ \pm}$can be decomposed into two maps if $n-k$ is even.

In $\S 5$, we prove exact sequences in homology, which are the key steps for calculating $H_{*}\left(M_{n, k}\right)$ by induction. Theorems 5.6 and $5.6^{\circ}$ are the main theorems of the section.

In $\S 6$, we first prove the properties which $f_{n, k}^{ \pm}: H_{*}\left(M_{n, k}\right) \rightarrow H_{*}\left(M_{n, k \pm 1}\right)$ satisfy, where $f_{n, k}^{ \pm}$are defined in $\S 3$. We do this in Theorem 6.3.

Having proved Theorem 6.3, we can apply Theorems 5.6 and $5.6^{\circ}$ to $H_{*}\left(M_{n, k}\right)$ and we establish recurrence relations for $P S(n, k)$. We do this in Theorem 6.7. Finally we solve the recurrence relations in Theorem 6.8. In particular, we have Theorems B and C.

\section{NotATIONS AND PRELIMINARIES}

In this section we define a map $\pi_{n, r}: M_{n, r} \rightarrow S^{1}(r>0)$ and prove that it is a fiber bundle with singular fibers. Then we prepare some notations which are used in later sections.

For an element $\left(u_{1}, \ldots, u_{n}\right) \in M_{n, r}(r \geq 0)$, we write coordinates of $u_{i}$ as follows: As in (1.3), we set $u_{1}=\left(\frac{r}{2}, 0\right)$ and $u_{2}=\left(-\frac{r}{2}, 0\right)$. On the other hand, we set $u_{i}=\left(x_{i}, y_{i}\right)$ for $3 \leq i \leq n$. When $r>0$, we also parameterize $M_{n, r}$ by parameters $\left\{\left(\theta_{1}, \ldots, \theta_{n}\right)\right\}$, where $\theta_{i}$ denotes the counter-clockwise angle from $\overrightarrow{u_{i} u_{i-1}}$ to $\overrightarrow{u_{i} u_{i+1}}$. (See Figure 1.)

We define $\pi_{n, r}: M_{n, r} \rightarrow S^{1}(r>0)$ by $\pi_{n, r}\left(\theta_{1}, \ldots, \theta_{n}\right)=\theta_{2}$. In order to study the singular fibers of $\pi_{n, r}$, we first consider the case of $r \in \mathbf{N}$. For such $r$, there exists a unique $\phi_{r} \in(0, \pi)$ so that if $\left(u_{1}, \ldots, u_{n}\right) \in \pi_{n, r}^{-1}\left(\phi_{r}\right)$, then $\left|u_{3}-u_{1}\right|=r$. For 
later convenience, we write $\phi_{r}$ as $\psi_{r}$ when $r$ is odd, and $\omega_{r}$ when $r$ is even. Then the structure of $\pi_{n, r}$ is given by the following:

\section{Proposition 2.1.}

(I) The case $n=2 m+1$ :

(i) The singular fibers of $\pi_{2 m+1,2 i+1}$ are given by $\pi_{2 m+1,2 i+1}^{-1}\left(\psi_{2 i+1}\right)$ and $\pi_{2 m+1,2 i+1}^{-1}\left(-\psi_{2 i+1}\right)(0 \leq i \leq m-2)$. Hence we have homeomorphisms: $\pi_{2 m+1,2 i+1}^{-1}\left(\left(-\psi_{2 i+1}, \psi_{2 i+1}\right)\right) \cong\left(-\psi_{2 i+1}, \psi_{2 i+1}\right) \times M_{2 m, 2 i}$, $\pi_{2 m+1,2 i+1}^{-1}\left(\left(\psi_{2 i+1}, 2 \pi-\psi_{2 i+1}\right)\right) \cong\left(\psi_{2 i+1}, 2 \pi-\psi_{2 i+1}\right) \times M_{2 m, 2 i+2}$.

(In what follows, we give only the singular fibers and omit such homeomorphisms.)

(ii) The singular fibers of $\pi_{2 m+1,2 m-1}$ are given by $\pi_{2 m+1,2 m-1}^{-1}\left(\psi_{2 m-1}\right)$ and $\pi_{2 m+1,2 m-1}^{-1}\left(-\psi_{2 m-1}\right)$.

(iii) The singular fibers of $\pi_{2 m+1,2 i}$ are given by $\pi_{2 m+1,2 i}^{-1}(0)$ and $\pi_{2 m+1,2 i}^{-1}(\pi)$ $(1 \leq i \leq m-1)$.

(II) The case $n=2 m$ :

(iv) The singular fibers of $\pi_{2 m, 2 i}$ are given by $\pi_{2 m, 2 i}^{-1}\left(\omega_{2 i}\right)$ and $\pi_{2 m, 2 i}^{-1}\left(-\omega_{2 i}\right)$ $(1 \leq i \leq m-2)$.

(v) The singular fibers of $\pi_{2 m, 2 m-2}$ are given by $\pi_{2 m, 2 m-2}^{-1}\left(\omega_{2 m-2}\right)$ and $\pi_{2 m, 2 m-2}^{-1}\left(-\omega_{2 m-2}\right)$.

(vi) The singular fibers of $\pi_{2 m, 2 i+1}$ are given by $\pi_{2 m, 2 i+1}^{-1}(0)$ and $\pi_{2 m, 2 i+1}^{-1}(\pi)$ $(1 \leq i \leq m-2)$.

Next we study the singular fibers of $\pi_{n, r}$ for $r \notin \mathbf{N}$. To do so, we generalize the definition of $\psi_{2 i+1}$ and $\omega_{2 i}$ to $\psi_{r}$ and $\omega_{r}$ as follows. For $r \notin \mathbf{N}$, we define $\psi_{r} \in$ $(0, \pi)$ to satisfy the following property: If $\left(u_{1}, \ldots, u_{n}\right) \in \pi_{n, r}^{-1}\left(\psi_{r}\right)$, then $\left|u_{3}-u_{1}\right|$ is odd. (Of course it is the unique odd number which is contained in $(r-1, r+1)$.) Similarly for $r \notin \mathbf{N}$, we define $\omega_{r} \in(0, \pi)$ to satisfy the following property: If $\left(u_{1}, \ldots, u_{n}\right) \in \pi_{n, r}^{-1}\left(\omega_{r}\right)$, then $\left|u_{3}-u_{1}\right|$ is even.

Then we have the following proposition for $r \notin \mathbf{N}$.

\section{Proposition 2.2.}

(i) The singular fibers of $\pi_{2 m+1, r}$ are given by $\pi_{2 m+1, r}^{-1}\left(\psi_{r}\right)$ and $\pi_{2 m+1, r}^{-1}\left(-\psi_{r}\right)$.

(ii) The singular fibers of $\pi_{2 m, r}$ are given by $\pi_{2 m, r}^{-1}\left(\omega_{r}\right)$ and $\pi_{2 m, r}^{-1}\left(-\omega_{r}\right)$.

The proofs of Propositions 2.1 and 2.2 are elementary. So we indicate only the proof of Proposition 2.1. We define $f: M_{n, r} \rightarrow \mathbf{R}$ by $f\left(u_{1}, \ldots, u_{n}\right)=\left|u_{3}-u_{1}\right|^{2}$. We need to find the critical points of $f$. In order to do so, we recall the result concerning the smoothness of $M_{n, r}(r>0)$. In the following proposition, to say " $x$ is a singular point of $M_{n, r}$ " means that the Jacobian matrix of polynomial functions over $\mathbf{R}$, whose locus of common zeros is $M_{n, r}$, is not of maximal rank at $x$.

Proposition 2.3 ([2], [5]).

(i) $M_{2 m+1, r}$ is a smooth manifold of dimension $2 m-2$ except for the case of $r=2 i(1 \leq i \leq m-1)$, in which case $\left(u_{1}, \ldots, u_{2 m+1}\right)$ is a singular point iff all of the $u_{i}$ lie on the $x$-axis, i.e., the line determined by $u_{1}$ and $u_{2}$.

(ii) $M_{2 m, r}$ is a smooth manifold of dimension $2 m-3$ except for the case of $r=$ $2 i+1(0 \leq i \leq m-2)$, in which case $\left(u_{1}, \ldots, u_{2 m}\right)$ is a singular point iff all of the $u_{i}$ lie on the $x$-axis. 
Now the critical points of $f$ are given by the following lemma:

Lemma 2.4. (i) $\left(u_{1}, \ldots, u_{2 m+1}\right) \in M_{2 m+1,2 i+1}$ is a critical point of $f$ iff $f\left(u_{1}, \ldots, u_{2 m+1}\right)=(2 i)^{2},(2 i+1)^{2}$, or $(2 i+2)^{2}$.

(ii) A smooth point of $\left(u_{1}, \ldots, u_{2 m+1}\right) \in M_{2 m+1,2 i}$ is a critical point of $f$ iff $f\left(u_{1}, \ldots, u_{2 m+1}\right)=(2 i-1)^{2}$ or $(2 i+1)^{2}$.

(iii) $\left(u_{1}, \ldots, u_{2 m}\right) \in M_{2 m, 2 i}$ is a critical point of $f$ iff $f\left(u_{1}, \ldots, u_{2 m}\right)=$ $(2 i-1)^{2},(2 i)^{2}$, or $(2 i+1)^{2}$.

(iv) A smooth point of $\left(u_{1}, \ldots, u_{2 m}\right) \in M_{2 m, 2 i+1}$ is a critical point of $f$ iff $f\left(u_{1}, \ldots, u_{2 m+1}\right)=(2 i)^{2}$ or $(2 i+2)^{2}$.

We see that Proposition 2.1 follows easily from the facts in Morse theory applied to Lemma 2.4.

Proof of Lemma 2.4. We define

$$
\begin{aligned}
f_{1}\left(x_{1}, y_{1}, \ldots, x_{n}, y_{n}\right) & =\left(x_{1}-x_{2}\right)^{2}+\left(y_{1}-y_{2}\right)^{2}-r^{2}, \\
f_{i}\left(x_{1}, y_{1}, \ldots, x_{n}, y_{n}\right) & =\left(x_{i}-x_{i+1}\right)^{2}+\left(y_{i}-y_{i+1}\right)^{2}-1(2 \leq i \leq n-1), \\
f_{n}\left(x_{1}, y_{1}, \ldots, x_{n}, y_{n}\right) & =\left(x_{n}-x_{1}\right)^{2}+\left(y_{n}-y_{1}\right)^{2}-1 .
\end{aligned}
$$

By $\left[9\right.$, Proposition 2.7], we see that a smooth point $\left(a_{1}, b_{1}, \ldots, a_{n}, b_{n}\right) \in\left\{\left(x_{1}, y_{1}, \ldots\right.\right.$, $\left.\left.x_{n}, y_{n}\right) \in \mathbf{R}^{2 n}: f_{i}\left(x_{1}, y_{1}, \ldots, x_{n}, y_{n}\right)=0(1 \leq i \leq n)\right\}$ is a critical point of $f$ iff $\operatorname{grad} f$ is spanned by $\left\{\operatorname{grad} f_{1}, \ldots, \operatorname{grad} f_{n}\right\}$ at this point. By using this fact, we can prove Lemma 2.4 easily.

Remark 2.5. As

$$
\pi_{2 m+1,2 m-1}\left(M_{2 m+1,2 m-1}\right)=\left[-\psi_{2 m-1}, \psi_{2 m-1}\right]
$$

and

$$
\pi_{2 m, 2 m-2}\left(M_{2 m, 2 m-2}\right)=\left[-\omega_{2 m-2}, \omega_{2 m-2}\right],
$$

the facts in Morse theory applied to Lemma 2.4 (i) and (iii) tell us that $M_{n, n-2}$ is homeomorphic to $S^{n-3}(n \geq 4)$.

Remark 2.6. We note that Proposition 2.3 supports the truth of Propositions 2.1 and 2.2. In fact, for example, we consider the case of $\pi_{2 m+1,2 i+1}: M_{2 m+1,2 i+1} \rightarrow S^{1}$. For each $\alpha \in S^{1}$, think of $\pi_{2 m+1,2 i+1}^{-1}(\alpha)$ as an element of $M_{2 m, s}$ by corresponding $\left(u_{1}, \ldots, u_{2 m+1}\right) \in \pi_{2 m+1,2 i+1}^{-1}(\alpha)$ to $\left(u_{1}, u_{3}, \ldots, u_{2 m+1}\right) \in M_{2 m, s}$, where we regard the line $\overline{u_{1} u_{3}}$ as the fixed line, and we set $s=\left|u_{3}-u_{1}\right|$.

If $\alpha \neq \pm \psi_{2 i+1}$, then Proposition 2.3 tells us that $\pi_{2 m+1,2 i+1}^{-1}(\alpha)$ is a smooth manifold. On the other hand, if $\alpha= \pm \psi_{2 i+1}$, then $\pi_{2 m+1,2 i+1}^{-1}(\alpha)$ has singular points. These facts indicate the truth of Propositions 2.1 and 2.2.

Proof of Theorem A. By applying Proposition 2.1 to $M_{2 m+1,2 i+1}$, and identifying $\pi_{2 m+1,2 i+1}^{-1}(\alpha)$ with $M_{2 m, s}$ as in Remark 2.6, we have homeomorphisms $M_{2 m, 2 i} \rightarrow$ $M_{2 m, s}$ for $2 i \leq s<2 i+1$. Similarly we have homeomorphisms $M_{2 m, 2 i} \rightarrow M_{2 m, s}$ for $2 i-1<s \leq 2 i$. Hence we have homeomorphisms $M_{2 m, 2 i} \rightarrow M_{2 m, s}$ for $2 i-1<$ $s<2 i+1$. 2 .

Similarly we have homeomorphisms $M_{2 m+1,2 i+1} \rightarrow M_{2 m+1, s}$ for $2 i<s<2 i+$ 


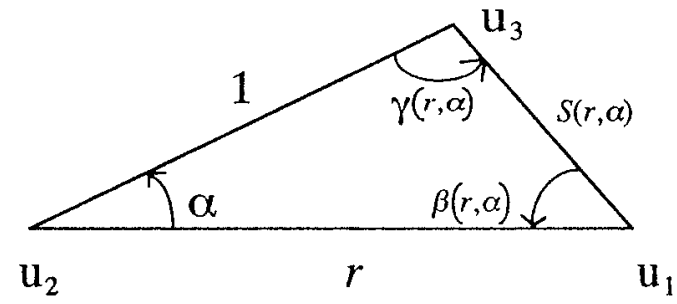

Figure 2

In $\S 3$, we construct the homeomorphisms in the proof of Theorem A more explicitly. In order to do so, we need some more notations, which we define in the rest of this section.

(1) Recall that we have a map $\pi_{n, r}: M_{n, r} \rightarrow S^{1}(r>0)$ given by $\pi_{n, r}\left(\theta_{1}, \ldots, \theta_{n}\right)$ $=\theta_{2}$. As usual, we parametrize $S^{1}$ by the parameter $\alpha$ of counter-clockwise angle. For $\alpha \in S^{1}$, take an element $\left(\theta_{1}, \alpha, \theta_{3}, \ldots, \theta_{n}\right) \in \pi_{n, r}^{-1}(\alpha)$ (thus we write $\theta_{2}$ as $\alpha$ ), and think of the triangle with vertices $\left(u_{1}, u_{2}, u_{3}\right)$. If $u_{1} \neq u_{3}$, then we define $\beta(r, \alpha), \gamma(r, \alpha)$ and $s(r, \alpha)$ as follows.

$\beta(r, \alpha)$ : the counter-clockwise angle from $\overrightarrow{u_{1} u_{3}}$ to $\overrightarrow{u_{1} u_{2}}$.

$\gamma(r, \alpha)$ : the counter-clockwise angle from $\overrightarrow{u_{3} u_{2}}$ to $\overrightarrow{u_{3} u_{1}}$.

$s(r, \alpha)$ : the distance $\left|u_{3}-u_{1}\right|$.

(See Figure 2.)

Concerning $\beta(r, \alpha)$ and $\gamma(r, \alpha)$, we can easily prove the following properties, which are used in later sections.

\section{Lemma 2.7.}

(i) $\beta(r, \alpha)$ and $\gamma(r, \alpha)$ are continuous except for the point where $(r, \alpha)=(1,0)$. Moreover we have the following:

(a) For $r>1$, we have $\lim _{\alpha \rightarrow 0} \beta(r, \alpha)=0$ and $\lim _{\alpha \rightarrow 0} \gamma(r, \alpha)=\pi$.

(b) For $r<1$, we have $\lim _{\alpha \rightarrow 0} \beta(r, \alpha)=\pi$ and $\lim _{\alpha \rightarrow 0} \gamma(r, \alpha)=0$.

(ii) $\beta(r, \alpha)$ and $\gamma(r, \alpha)$ are not continuous at $(r, \alpha)=(1,0)$. In fact we have the following:

(c) $\lim _{\alpha \rightarrow+0} \beta(1, \alpha)=\lim _{\alpha \rightarrow+0} \gamma(1, \alpha)=\frac{\pi}{2}$,

(d) $\lim _{\alpha \rightarrow-0} \beta(1, \alpha)=\lim _{\alpha \rightarrow-0} \gamma(1, \alpha)=\frac{3 \pi}{2}$.

(2) As in Remark 2.6, we can identify $\pi_{n, r}^{-1}(\alpha)$ with $M_{n-1, s(r, \alpha)}$ if $(r, \alpha) \neq(1,0)$, i.e., $s(r, \alpha) \neq 0$. We write down this identification $\mu(r, \alpha): \pi_{n, r}^{-1}(\alpha) \stackrel{\simeq}{\longrightarrow} M_{n-1, s(r, \alpha)}$ more explicitly. Take $\left(\theta_{1}, \theta_{2}, \ldots, \theta_{n}\right) \in \pi_{n, r}^{-1}(\alpha)$. By (1), the triangle $\left(u_{1}, u_{2}, u_{3}\right)$ can be written as $(\beta(r, \alpha), \alpha, \gamma(r, \alpha))$. Hence the $(n-1)$-gon $\left(u_{1}, u_{3}, u_{4}, \ldots, u_{n}\right)$ can be written as $\left(\theta_{1}-\beta(r, \alpha), \theta_{3}-\gamma(r, \alpha), \theta_{4}, \ldots, \theta_{n}\right)$. Let us write this element as $\left(\bar{\theta}_{1}, \bar{\theta}_{3}, \theta_{4}, \ldots, \theta_{n}\right)$. As $\left|u_{3}-u_{1}\right|=s(r, \alpha)$, we can regard $\left(\bar{\theta}_{1}, \bar{\theta}_{3}, \theta_{4}, \ldots, \theta_{n}\right)$ as an element of $M_{n-1, s(r, \alpha)}$, with the fixed line $\overline{u_{1} u_{3}}$. Hence we can define a homeomorphism $\mu(r, \alpha)$ by

$$
\mu(r, \alpha)\left(\theta_{1}, \alpha, \theta_{3}, \theta_{4}, \ldots, \theta_{n}\right)=\left(\bar{\theta}_{1}, \bar{\theta}_{3}, \theta_{4}, \ldots, \theta_{n}\right) .
$$

(3) So far we have treated only $M_{n, r}$ with $r>0$. Concerning $M_{n, 0}$, we have the following: 


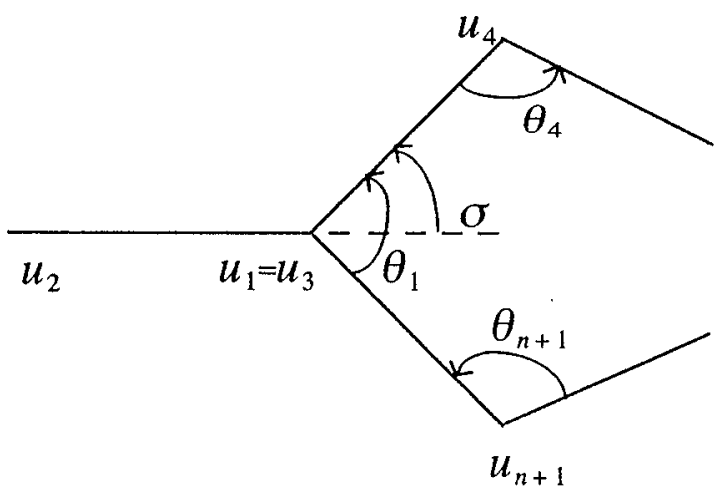

FigURE 3

Lemma 2.9. We have homeomorphisms

$$
\begin{aligned}
\mu(1,0) & : \pi_{n+1,1}^{-1}(0) \rightarrow M_{n, 0}, \\
\eta & : M_{n, 0} \rightarrow S^{1} \times M_{n-1,1} .
\end{aligned}
$$

Proof. For $\left(u_{1}, u_{2}, \ldots, u_{n+1}\right) \in \pi_{n+1,1}^{-1}(0)$, we set $\mu(1,0)\left(u_{1}, u_{2}, \ldots, u_{n+1}\right)=$ $\left(u_{1}, u_{3}, u_{4}, \ldots, u_{n+1}\right)$. As $u_{1}=u_{3}$, we can assume that $\left(u_{1}, u_{3}, u_{4}, \ldots, u_{n+1}\right) \in$ $M_{n, 0}$.

For $\left(u_{1}, u_{2}, \ldots, u_{n}\right) \in M_{n, 0}$ (recall that $u_{1}=u_{2}=O$ for such an element), we define $\pi_{n, 0}: M_{n, 0} \rightarrow S^{1}$ by $\pi_{n, 0}\left(\left(u_{1}, \ldots, u_{n}\right)\right)=u_{3}$. We identify $u_{3}$ with its counter-clockwise angle $\sigma$ from $\overrightarrow{O e_{1}}$ to $\overrightarrow{O u_{3}}$, where we set $e_{1}=\left(\begin{array}{l}1 \\ 0\end{array}\right)$.

If we form $\left(u_{1}, u_{3}, \ldots, u_{n}\right)$ from $\left(u_{1}, u_{2}, \ldots, u_{n}\right)$, then we can assume that $\left(u_{1}, u_{3}, \ldots, u_{n}\right) \in M_{n-1,1}$ with the fixed line $\overline{u_{1} u_{3}}$. (A more explicit identification is as follows: By rotating $\left(u_{1}, u_{2}, \ldots, u_{n}\right)$ by $\pi-\sigma$ around $O$, we have an element $\left(O, O,-e_{1}, u_{4}^{\prime}, \ldots, u_{n}^{\prime}\right)$. If we form $\left(\frac{1}{2} e_{1},-\frac{1}{2} e_{1}, u_{4}^{\prime}+\frac{1}{2} e_{1}, \ldots, u_{n}^{\prime}+\frac{1}{2} e_{1}\right)$, we can regard it as an element of $M_{n-1,1}$.)

Then we define $\eta$ by $\eta\left(u_{1}, u_{2}, \ldots, u_{n}\right)=\left(\sigma,\left(\frac{1}{2} e_{1},-\frac{1}{2} e_{1}, u_{4}^{\prime}+\frac{1}{2} e_{1}, \ldots, u_{n}^{\prime}+\frac{1}{2} e_{1}\right)\right)$.

Remark 2.10. We note that $\mu(1,0)^{-1} \cdot \eta^{-1}: S^{1} \times M_{n-1,1} \rightarrow \pi_{n+1,1}^{-1}(0)$ is given as follows: We write an element of $S^{1} \times M_{n-1,1}$ as $\left(\sigma,\left(\theta_{1}, \theta_{4}, \ldots, \theta_{n+1}\right)\right)$. Then

$$
\mu(1,0)^{-1} \cdot \eta^{-1}\left(\sigma,\left(\theta_{1}, \theta_{4}, \ldots, \theta_{n+1}\right)\right)=\left(\pi-\sigma+\theta_{1}, 0, \pi+\sigma, \theta_{4}, \ldots, \theta_{n+1}\right) .
$$

(See Figure 3.)

\section{Deformations of POLYGONS}

As indicated in $\S 2$, the purpose of this section is to prove the following:

Theorem 3.1. (I) For $m \geq 3,1 \leq k \leq n-2$ and $n-k \equiv 0 \bmod 2$, we have a map

$$
f_{n}^{k}: M_{n, k} \times(k-1, k+1) \rightarrow\left(S^{1}\right)^{n-1}
$$

which satisfies the following properties (i)-(iv).

For $r \in(k-1, k+1)$, we define $f_{n, r}^{k}: M_{n, k} \rightarrow\left(S^{1}\right)^{n-1}$ to be the restriction of $f_{n}^{k}$ on $M_{n, k} \times\{r\}$. 
(i) $f_{n, r}^{k}$ is injective and $\operatorname{Im} f_{n, r}^{k}=M_{n, r}$. Hence $f_{n, r}^{k}: M_{n, k} \rightarrow M_{n, r}$ is a homeomorphism.

(ii) $f_{n, k}^{k}=i d$.

(iii) $\lim _{r \rightarrow k-1+0} f_{n, r}^{k}$ and $\lim _{r \rightarrow k+1-0} f_{n, r}^{k}$ exist. We set

$$
f_{n, k}^{-}=\lim _{r \rightarrow k-1+0} f_{n, r}^{k} \quad \text { and } \quad f_{n, k}^{+}=\lim _{r \rightarrow k+1-0} f_{n, r}^{k} .
$$

(iv) By (iii), we see that $f_{n}^{k}: M_{n, k} \times(k-1, k+1) \rightarrow\left(S^{1}\right)^{n-1}$ is extendable to a map $f_{n}^{k}: M_{n, k} \times[k-1, k+1] \rightarrow\left(S^{1}\right)^{n-1}$. We require that the latter map is continuous.

(II) Moreover, when $n \equiv 0 \bmod 2$, we have a map

$$
f_{n}^{0}: M_{n, 0} \times[0,1) \rightarrow\left(S^{1}\right)^{n-1}
$$

which satisfies properties similar to those in (I).

For the rest of this section, we prove Theorem 3.1. To avoid confusion, we set

$$
\begin{cases}f_{2 m+1}^{2 i+1}=\tau_{2 m+1}^{2 i+1}: M_{2 m+1,2 i+1} \times(2 i, 2 i+2) \rightarrow\left(S^{1}\right)^{2 m}, & k=2 i+1, \\ f_{2 m}^{2 i}=\rho_{2 m}^{2 i}: M_{2 m, 2 i} \times(2 i-1,2 i+1) \rightarrow\left(S^{1}\right)^{2 m-1}, & k=2 i .\end{cases}
$$

When the number of vertices $n=2 m+1$ or $n=2 m$ is clearly understood, we drop these indices from $\tau_{2 m+1}^{2 i+1}$ or $\rho_{2 m}^{2 i}$. Thus $f_{n, r}^{k}: M_{n, k} \rightarrow M_{n, r}$ in Theorem 3.1 is written as

$$
\begin{cases}\tau_{r}^{2 i+1}: M_{2 m+1,2 i+1} \rightarrow M_{2 m+1, r}, \quad r \in(2 i, 2 i+2), & k=2 i+1, \\ \rho_{r}^{2 i}: M_{2 m, 2 i} \rightarrow M_{2 m, r}, \quad r \in(2 i-1,2 i+1), & k=2 i .\end{cases}
$$

Before beginning the proof, we explain the essential idea for constructing $\tau_{r}^{2 i+1}$ and $\rho_{r}^{2 i}$. As their ideas are similar, we explain for $\tau_{r}^{2 i+1}$. Take an element $\left(\theta_{1}, \theta_{2}, \ldots, \theta_{2 m+1}\right) \in M_{2 m+1,2 i+1}$. As in $\S 2(2)$, we separate it into a triangle $(\beta(2 i+1, \alpha), \alpha, \gamma(2 i+1, \alpha))$ and a $2 m$-gon $\left(\bar{\theta}_{1}, \bar{\theta}_{3}, \theta_{4}, \ldots, \theta_{2 m+1}\right)$, where $\bar{\theta}_{1}=$ $\theta_{1}-\beta(2 i+1, \alpha), \bar{\theta}_{3}=\theta_{3}-\gamma(2 i+1, \alpha)$.

(i) First deform the triangle to $\left(\beta\left(r, \alpha^{\prime}\right), \alpha^{\prime}, \gamma\left(r, \alpha^{\prime}\right)\right)$, i.e., a triangle with the length of the fixed line being $r$ ( $\alpha^{\prime}$ is chosen suitably). Note that the length of the oblique side of this new triangle is equal to $s\left(r, \alpha^{\prime}\right)$.

(ii) Next think of $\left(\bar{\theta}_{1}, \bar{\theta}_{3}, \theta_{4}, \ldots, \theta_{2 m+1}\right) \in \pi_{2 m+1,2 i+1}^{-1}(\alpha)$ as an element of $M_{2 m, s(2 i+1, \alpha)}$ by $\mu(r, \alpha)$ (cf. $\left.\S 2(2)\right)$. Then deform $\left(\bar{\theta}_{1}, \bar{\theta}_{3}, \theta_{4}, \ldots, \theta_{2 m+1}\right)$ to $\left(\bar{\theta}_{1}^{\prime}, \bar{\theta}_{3}^{\prime}, \theta_{4}^{\prime}, \ldots, \theta_{2 m+1}^{\prime}\right)$, so that the length of the fixed line of this new $2 m$-gon, i.e., $\overline{u_{1} u_{3}}$, is equal to $s\left(r, \alpha^{\prime}\right)$.

(iii) Finally attach $\left(\beta\left(r, \alpha^{\prime}\right), \alpha^{\prime}, \gamma\left(r, \alpha^{\prime}\right)\right)$ and $\left(\bar{\theta}_{1}^{\prime}, \bar{\theta}_{3}^{\prime}, \theta_{4}^{\prime}, \ldots, \theta_{2 m+1}^{\prime}\right)$ along the lines of length $s\left(r, \alpha^{\prime}\right)$, i.e., $\overline{u_{1} u_{3}}$. Then we get a new $(2 m+1)$-gon

$$
\left(\bar{\theta}_{1}^{\prime}+\beta\left(r, \alpha^{\prime}\right), \alpha^{\prime}, \bar{\theta}_{3}^{\prime}+\gamma\left(r, \alpha^{\prime}\right), \theta_{4}^{\prime}, \ldots, \theta_{2 m+1}^{\prime}\right) .
$$

We denote this $(2 m+1)$-gon as $\tau_{r}^{2 i+1}\left(\theta_{1}, \theta_{2}, \ldots, \theta_{2 m+1}\right)$.

Hereafter, we use the following notations.

Notation 3.3. (i) If $\tau_{r}^{2 i+1}$ is constructed as in Theorem 3.1 (I), then we define $\tau_{r_{2}}^{r_{1}}: M_{2 m+1, r_{1}} \rightarrow M_{2 m+1, r_{2}}\left(2 i<r_{1}, r_{2}<2 i+2\right)$ by $\tau_{r_{2}}^{r_{1}}=\tau_{r_{2}}^{2 i+1} \cdot\left(\tau_{r_{1}}^{2 i+1}\right)^{-1}$.

(ii) If $\rho_{r}^{0}$ or $\rho_{r}^{2 i}$ is constructed as in Theorem 3.1 (I) or (II), then we define $\rho_{r_{2}}^{r_{1}}$ : $M_{2 m, r_{1}} \rightarrow M_{2 m, r_{2}} \quad\left(0 \leq r_{1}, r_{2}<1\right)$ or $\rho_{r_{2}}^{r_{1}}: M_{2 m, r_{1}} \rightarrow M_{2 m, r_{2}} \quad(2 i-1<$ $\left.r_{1}, r_{2}<2 i+1\right)$ in the same way as we defined $\tau_{r_{2}}^{r_{1}}$. 
Now we prove Theorem 3.1 by induction on $n$, where $n$ is the number of vertices of polygons, i.e., $n=2 m+1$ or $2 m$. For the initial condition of the induction, it is clear that we have a family of homeomorphisms $\tau^{1}: M_{3,1} \times(0,2) \rightarrow\left(S^{1}\right)^{2}$.

(A) Assume that $\tau^{2 i+1}: M_{2 m+1,2 i+1} \times(2 i, 2 i+2) \rightarrow\left(S^{1}\right)^{2 m}$ are constructed for $0 \leq i \leq m-1$.

We need to construct $\rho^{0}: M_{2 m+2,0} \times[0,1) \rightarrow\left(S^{1}\right)^{2 m+1}, \rho^{2}: M_{2 m+2,2} \times(1,3) \rightarrow$ $\left(S^{1}\right)^{2 m+1}$, and $\rho^{2 i}: M_{2 m+2,2 i} \times(2 i-1,2 i+1) \rightarrow\left(S^{1}\right)^{2 m+1}$ for $2 \leq i \leq m$. In order to do so, it suffices to construct

$$
\begin{aligned}
& \rho_{r}^{0}: M_{2 m+2,0} \rightarrow M_{2 m+2, r}, \quad r \in[0,1), \\
& \rho_{r}^{2}: M_{2 m+2,2} \rightarrow M_{2 m+2, r}, \quad r \in(1,3),
\end{aligned}
$$

and

$$
\rho_{r}^{2 i}: M_{2 m+2,2 i} \rightarrow M_{2 m+2, r}, \quad r \in(2 i-1,2 i+1) .
$$

(i) Constructions of $\rho_{r}^{2 i}$ for $2 \leq i \leq m$. First we deform $(\beta(2 i, \alpha), \alpha, \gamma(2 i, \alpha))$. In order to do so, we only need to designate how $\alpha$ changes as $r$ moves, i.e. to designate a function $g_{r}^{2 i}:[0,2 \pi] \rightarrow[0,2 \pi]$. We consider the properties which $g_{r}^{2 i}$ should satisfy.

As $\rho_{r}^{2 i}$ should satisfy $\rho_{2 i}^{2 i}=i d, g_{r}^{2 i}$ should satisfy

$$
g_{2 i}^{2 i}=i d \text {. }
$$

Actually $g_{r}^{2 i}$ should satisfy one more property. Recall that $\pi_{2 m+2, r}^{-1}\left(\omega_{r}\right)$ is a singular fiber for $2 i-1<r<2 i+1$ by Propositions 2.1 and 2.2. Think of the situation that $u_{1}$ moves with $u_{2}$ fixed, i.e., the length $r$ of the fixed line moves away from $2 i$. In this situation, the singular fiber $\pi_{2 m+2,2 i}^{-1}\left(\omega_{2 i}\right)$ should be deformed to a singular fiber $\pi_{2 m+2, r}^{-1}\left(\omega_{r}\right)$. Equivalently, in the course of deformation of the triangle $\left(\beta\left(2 i, \omega_{2 i}\right), \omega_{2 i}, \gamma\left(2 i, \omega_{2 i}\right)\right)$, the length of the oblique side $\left(=s\left(r, g_{r}^{2 i}\left(\omega_{2 i}\right)\right)\right)$ should always satisfy $s\left(r, g_{r}^{2 i}\left(\omega_{2 i}\right)\right)=2 i$. And, by the definition of $\omega_{r}$, this is equivalent to

$$
g_{r}^{2 i}\left(\omega_{2 i}\right)=\omega_{r} \quad(2 i-1<r<2 i+1)
$$

Now it is natural that we define $g_{r}^{2 i}$, which satisfies (3.4) and (3.5), by the following manner: Think of a graph $\omega_{r}$ in the $\{r\} \times\{\alpha\}$ plane. If $r$ moves in $(2 i-1,2 i+1)$, then $\omega_{r}$ is a decreasing function, so

$$
\lim _{r \rightarrow 2 i-1+0} \omega_{r}=\pi, \quad \lim _{r \rightarrow 2 i+1-0} \omega_{r}=0 .
$$

An element $\alpha \in[0, \pi]$ can be written as $\alpha=\lambda \omega_{2 i}$ or $\alpha=\lambda \omega_{2 i}+(1-\lambda) \pi(0 \leq \lambda \leq 1)$. So we define $g_{r}^{2 i}(\alpha)$ to be the internal dividing point of $\left[0, \omega_{r}\right]$ or $\left[\omega_{r}, \pi\right]$ which preserves $\lambda$, i.e., for $\alpha \in[0, \pi]$,

$$
g_{r}^{2 i}(\alpha)= \begin{cases}\lambda \omega_{r} & \text { if } \alpha=\lambda \omega_{2 i}(0 \leq \lambda \leq 1), \\ \lambda \omega_{r}+(1-\lambda) \pi & \text { if } \alpha=\lambda \omega_{2 i}+(1-\lambda) \pi \quad(0 \leq \lambda \leq 1) .\end{cases}
$$

Finally we define $g_{r}^{2 i}(-\alpha)$ for $\alpha \in[0, \pi]$ by

$$
g_{r}^{2 i}(-\alpha)=-g_{r}^{2 i}(\alpha) \text { for } \alpha \in[0, \pi] .
$$

Thus we have completed the definition of $g_{r}^{2 i}$.

Now we deform a triangle $(\beta(2 i, \alpha), \alpha, \gamma(2 i, \alpha))$ to the triangle

$$
\left(\beta\left(r, g_{r}^{2 i}(\alpha)\right), g_{r}^{2 i}(\alpha), \gamma\left(r, g_{r}^{2 i}(\alpha)\right)\right)
$$


Next we deform $\left(\bar{\theta}_{1}, \bar{\theta}_{3}, \theta_{4}, \ldots, \theta_{2 m+2}\right)$. Note that $\left|u_{3}-u_{1}\right|$ of this $(2 m+1)$-gon is equal to $s(2 i, \alpha)$. On the other hand, the oblique side of the deformed triangle has length $s\left(r, g_{r}^{2 i}(\alpha)\right)$. So we need to deform $\left(\bar{\theta}_{1}, \bar{\theta}_{3}, \theta_{4}, \ldots, \theta_{2 m+2}\right)$ so that the length of the fixed line $\left(=\left|u_{1}-u_{3}\right|\right)$ is equal to $s\left(r, g_{r}^{2 i}(\alpha)\right)$. Note that by the definition of $g_{r}^{2 i}$, we have

(a) If $2 i-1 \leq s(2 i, \alpha)<2 i$, then $2 i-1 \leq s\left(r, g_{r}^{2 i}(\alpha)\right)<2 i$.

(b) If $2 i<s(2 i, \alpha) \leq 2 i+1$, then $2 i<s\left(r, g_{r}^{2 i}(\alpha)\right) \leq 2 i+1$.

By the inductive hypothesis, we have a homeomorphism $\tau_{r_{2}}^{r_{1}}: M_{2 m+1, r_{1}} \rightarrow M_{2 m+1, r_{2}}$ for $2 i-1 \leq r_{1}, r_{2}<2 i$ or $2 i<r_{1}, r_{2} \leq 2 i+1$. Hence if $\alpha \neq \pm \omega_{2 i}$, we can deform $\left(\bar{\theta}_{1}, \bar{\theta}_{3}, \theta_{4}, \ldots, \theta_{2 m+2}\right)$ to

$$
\tau_{s\left(r, g_{r}^{2 i}(\alpha)\right)}^{s(2 i, \alpha)}\left(\bar{\theta}_{1}, \bar{\theta}_{3}, \theta_{4}, \ldots, \theta_{2 m+2}\right) \in M_{2 m+1, s\left(r, g_{r}^{2 i}(\alpha)\right)} .
$$

If $\alpha= \pm \omega_{2 i}$, we define the deformation of $M_{2 m+1,2 i}$ to itself by the identity map.

Finally we attach the deformed triangle and the deformed $(2 m+1)$-gon along the lines of length $s\left(r, g_{r}^{2 i}(\alpha)\right)$, i.e., $\overline{u_{1} u_{3}}$. Then we have the following definition of $\rho_{r}^{2 i}$.

$$
\begin{gathered}
\rho_{r}^{2 i}\left(\theta_{1}, \alpha, \theta_{3}, \ldots, \theta_{2 m+2}\right) \\
=\left\{\begin{array}{c}
\left(\beta\left(r, g_{r}^{2 i}(\alpha)\right), g_{r}^{2 i}(\alpha), \gamma\left(r, g_{r}^{2 i}(\alpha)\right)\right) \dot{+} \tau_{s\left(r, g_{r}^{2 i}(\alpha)\right)}^{s(2 i, \alpha}\left(\bar{\theta}_{1}, \bar{\theta}_{3}, \theta_{4}, \ldots, \theta_{2 m+2}\right) \\
\text { for } \alpha \neq \pm \omega_{2 i}, \\
\left(\beta\left(r, g_{r}^{2 i}(\alpha)\right), g_{r}^{2 i}(\alpha), \gamma\left(r, g_{r}^{2 i}(\alpha)\right)\right) \dot{+}\left(\bar{\theta}_{1}, \bar{\theta}_{3}, \theta_{4}, \ldots, \theta_{2 m+2}\right) \\
\text { for } \alpha= \pm \omega_{2 i},
\end{array}\right.
\end{gathered}
$$

where the symbol $\dot{+}$ is defined as follows: For example, for

$$
\left(\beta\left(r, g_{r}^{2 i}(\alpha)\right), g_{r}^{2 i}(\alpha), \gamma\left(r, g_{r}^{2 i}(\alpha)\right)\right) \dot{+} \tau_{s\left(r, g_{r}^{2 i}(\alpha)\right)}^{s(2 i, \alpha)}\left(\bar{\theta}_{1}, \bar{\theta}_{3}, \theta_{4}, \ldots, \theta_{2 m+2}\right),
$$

write $\tau_{s\left(r, g_{r}^{2 i}(\alpha)\right)}^{s(2 i, \alpha)}\left(\bar{\theta}_{1}, \bar{\theta}_{3}, \theta_{4}, \ldots, \theta_{2 m+2}\right)$ as $\left(\bar{\theta}_{1}^{\prime}, \bar{\theta}_{3}^{\prime}, \theta_{4}^{\prime}, \ldots, \theta_{2 m+2}^{\prime}\right)$. Then the above formula is understood as $\left(\bar{\theta}_{1}^{\prime}+\beta\left(r, g_{r}^{2 i}(\alpha)\right), g_{r}^{2 i}(\alpha), \bar{\theta}_{3}^{\prime}+\gamma\left(r, g_{r}^{2 i}(\alpha)\right), \theta_{4}^{\prime}, \ldots, \theta_{2 m+2}^{\prime}\right)$.

In the following we always use the symbol $\dot{+}$ in this sense.

Concerning (3.9), if we fix $r \in(2 i-1,2 i+1)$, then $\rho_{r}^{2 i}$ given in (3.9) is continuous at $\pi_{2 m+2, r}^{-1}\left( \pm \omega_{r}\right)$, because of the inductive hypothesis of Theorem 3.1 (I). By using Lemma 2.7 (i), we can easily prove that $\rho_{r}^{2 i}$ satisfies the conditions of Theorem 3.1 (I). This completes the constructions of $\rho_{r}^{2 i}(2 i-1<r<2 i+1)$ for $2 \leq i \leq m$.

(ii) Construction of $\rho_{r}^{2}$. In order to construct $\rho_{r}^{2}(1<r<3)$, note that $\rho_{r}^{2}(2 \leq$ $r<3$ ) can be constructed in the same way as in (i). So we need to construct $\rho_{r}^{2}(1<r \leq 2)$. If we follow the steps in (i) in order to construct $\rho_{r}^{2}(1<r \leq 2)$, we see that this deformation is not continuous as $r \rightarrow 1$ and $\alpha \rightarrow 0$. The essential reason for this is that $\beta(r, \alpha)$ and $\gamma(r, \alpha)$ are not continuous at $(r, \alpha)=(1,0)$ in the notation of Lemma 2.7. Hence we replace $g_{r}^{2 i}$ in (i) by a nicer function $G_{r}^{2}$, i.e., deform triangles $(\beta(2, \alpha), \alpha, \gamma(2, \alpha))$ more nicely.

For the same reason as in (3.4) and (3.5), we must have $G_{2}^{2}=i d$ and $G_{r}^{2}\left(\omega_{2}\right)=\omega_{r}$. Think of a graph $\omega_{r}$ in the $\{r\} \times\{\alpha\}$ plane. If $r$ moves in $(1,2]$, then $\omega_{r}$ is a decreasing function, so that $\lim _{r \rightarrow 1+0} \omega_{r}=\pi$. Choose a number, say $\frac{\pi}{3}$ which satisfies $0<\frac{\pi}{3}<\omega_{2}$, then think of a line $l_{0}$ in the $\{r\} \times\{\alpha\}$ plane, which contains the coordinates $(1,0)$ and $\left(2, \frac{\pi}{3}\right)$. We see that $l_{0}$ contains $\left(r, \frac{\pi}{3}(r-1)\right)$. An element $\alpha \in[0, \pi]$ can be written as $\alpha=\lambda \frac{\pi}{3}, \alpha=\lambda \frac{\pi}{3}+(1-\lambda) \omega_{2}$, or $\alpha=\lambda \omega_{2}+(1-\lambda) \pi(0 \leq$ 
$\lambda \leq 1)$. So let $G_{r}^{2}(\alpha)$ be the internal dividing point of $\left[0, \frac{\pi}{3}(r-1)\right],\left[\frac{\pi}{3}(r-1), \omega_{r}\right]$, or $\left[\omega_{r}, \pi\right]$, which preserves $\lambda$, i.e., for $\alpha \in[0, \pi]$,

$$
G_{r}^{2}(\alpha)= \begin{cases}\lambda \frac{\pi}{3}(r-1) & \text { if } \alpha=\lambda \frac{\pi}{3}(0 \leq \lambda \leq 1) \\ \lambda \frac{\pi}{3}(r-1)+(1-\lambda) \omega_{r} & \text { if } \alpha=\lambda \frac{\pi}{3}+(1-\lambda) \omega_{2}(0 \leq \lambda \leq 1) \\ \lambda \omega_{r}+(1-\lambda) \pi & \text { if } \alpha=\lambda \omega_{2}+(1-\lambda) \pi(0 \leq \lambda \leq 1)\end{cases}
$$

Then define $G_{r}^{2}(-\alpha)=-G_{r}^{2}(\alpha)$ for $\alpha \in[0, \pi]$.

Finally we define $\rho_{r}^{2}$ as in (3.9), i.e.,

$$
\begin{aligned}
& \rho_{r}^{2}(\left.\theta_{1}, \alpha, \theta_{3}, \ldots, \theta_{2 m+2}\right) \\
& \quad=\left\{\begin{aligned}
\left(\beta\left(r, G_{r}^{2}(\alpha)\right), G_{r}^{2}(\alpha), \gamma\left(r, G_{r}^{2}(\alpha)\right)\right) & \\
\dot{+} \tau_{s\left(r, G_{r}^{2}(\alpha)\right)}^{s(2, \alpha)}\left(\bar{\theta}_{1}, \bar{\theta}_{3}, \theta_{4}, \ldots, \theta_{2 m+2}\right) & \text { for } \alpha \neq \pm \omega_{2}, \\
\left(\beta\left(r, G_{r}^{2}(\alpha)\right), G_{r}^{2}(\alpha), \gamma\left(r, G_{r}^{2}(\alpha)\right)\right) \dot{+}\left(\bar{\theta}_{1}, \bar{\theta}_{3}, \theta_{4}, \ldots, \theta_{2 m+2}\right) & \text { for } \alpha= \pm \omega_{2} .
\end{aligned}\right.
\end{aligned}
$$

The fact that $\rho_{r}^{2}$ is continuous as $r \rightarrow 1$ and $\alpha \rightarrow 0$ can be checked easily by using the following fact, which tells us that $\left(\beta\left(r, G_{r}^{2}(\alpha)\right), G_{r}^{2}(\alpha), \gamma\left(r, G_{r}^{2}(\alpha)\right)\right)$ is continuous as $r \rightarrow 1$ and $\alpha \rightarrow 0$ : Assume that $|\alpha|$ is small. Then we can write $\alpha=\lambda \frac{\pi}{3}$, and $G_{r}^{2}(\alpha)=\lambda \frac{\pi}{3}(r-1)$. From the deformed triangle $\left(\beta\left(r, G_{r}^{2}(\alpha)\right), G_{r}^{2}(\alpha), \gamma\left(r, G_{r}^{2}(\alpha)\right)\right)$, we know that

$$
\sin ^{2} \beta\left(r, G_{r}^{2}(\alpha)\right)=\frac{\sin ^{2} \lambda \frac{\pi}{3}(r-1)}{r^{2}+1-2 r \cos \lambda \frac{\pi}{3}(r-1)} .
$$

Then we can prove that

$$
\lim _{r \rightarrow 1} \sin ^{2} \beta\left(r, G_{r}^{2}(\alpha)\right)=\frac{\lambda^{2} \pi^{2}}{9+\lambda^{2} \pi^{2}} .
$$

This completes the construction of $\rho_{r}^{2}(1<r<3)$.

Remark 3.12. Note that when $|\alpha|$ is small, we have constructed $G_{r}^{2}$ by the idea of a real version of "blowing-up."

(iii) Construction of $\rho_{r}^{0}$. In order to construct $\rho_{r}^{0}(0 \leq r<1)$, first we construct $\rho_{r}^{0} \quad\left(0 \leq r \leq \frac{1}{2}\right)$. Take an element of $M_{2 m+2,0}$ and by $\eta$ (cf. Lemma 2.9), regard it as an element of $S^{1} \times M_{2 m+1,1}$ and write it as $\left(\sigma,\left(\theta_{1}, \theta_{3}, \ldots, \theta_{2 m+2}\right)\right)$. Prepare a triangle $(\beta(r, \sigma), \sigma, \gamma(r, \sigma))$. (Note that the length of the oblique side of this triangle is equal to $s(r, \sigma)$.) Deform $\left(\theta_{1}, \theta_{3}, \ldots, \theta_{2 m+2}\right) \in M_{2 m+1,1}$ to $\tau_{s(r, \alpha)}^{1}\left(\theta_{1}, \theta_{3}, \ldots, \theta_{2 m+2}\right)$. Finally attach this triangle and $(2 m+1)$-gon along the lines of length $s(r, \alpha)$. Thus

$$
\rho_{r}^{0}\left(\sigma,\left(\theta_{1}, \theta_{3}, \ldots, \theta_{2 m+2}\right)\right)=(\beta(r, \sigma), \sigma, \gamma(r, \sigma)) \dot{+} \tau_{s(r, \alpha)}^{1}\left(\theta_{1}, \theta_{3}, \ldots, \theta_{2 m+2}\right),
$$

for $0 \leq r \leq \frac{1}{2}$.

Next we define the deformation $\rho_{r}^{\frac{1}{2}}: M_{2 m+2, \frac{1}{2}} \rightarrow M_{2 m+2, r} \quad\left(\frac{1}{2} \leq r<1\right)$ in the same way as in $\rho_{r}^{2}(1<r \leq 2)$, i.e., by "blowing-up" along the line through $(1,0)$ and $\left(\frac{1}{2}, \frac{\pi}{3}\right)$. Then define $\rho_{r}^{0}\left(\frac{1}{2} \leq r<1\right)$ by $\rho_{r}^{0}=\rho_{r}^{\frac{1}{2}} \cdot \rho_{\frac{1}{2}}^{0}$. This completes the construction of $\rho_{r}^{0}(0 \leq r<1)$.

(B) Assume $\rho^{0}: M_{2 m, 0} \times[0,1) \rightarrow\left(S^{1}\right)^{2 m-1}$ and $\rho^{2 i}: M_{2 m, 2 i} \times(2 i-1,2 i+1)$ $\rightarrow\left(S^{1}\right)^{2 m-1}$ are constructed for $1 \leq i \leq m-1$. 
We need to construct $\tau^{2 i+1}: M_{2 m+1,2 i+1} \times(2 i, 2 i+2) \rightarrow\left(S^{1}\right)^{2 m}$ for $0 \leq i \leq m-1$. In order to do so, it suffices to construct

$$
\tau_{r}^{2 i+1}: M_{2 m+1,2 i+1} \rightarrow M_{2 m+1, r}, \quad r \in(2 i, 2 i+2) .
$$

We note that $\tau_{r}^{2 i+1}(1 \leq i \leq m-1)$ can be constructed in the same way as in (A)-(i) by taking $\psi_{r}$ instead of $\omega_{r}$ (as for $\psi_{r}$, see Propositions 2.1 and 2.2). Thus we need to construct only $\tau_{r}^{1}(0<r<2)$. In order to do so, we can construct $\tau_{r}^{1}$ independently for the two cases $0<r \leq 1$ and $1 \leq r<2$. But as the constructions for these two cases are similar, we construct $\tau_{r}^{1}$ only for $1 \leq r<2$.

By the same reason as in the construction of (A)-(ii), the essential part for which we must be careful in order to construct $\tau_{r}^{1}$ is the part for $\tau_{1+\epsilon}^{1}$ (where $\epsilon>0$ is small), i.e., a deformation of an element of $M_{2 m+1,1}$ to a $(2 m+1)$-gon whose fixed line has length slightly larger than 1 . We construct $\tau_{r}^{1}$ in the following way.

Fix a number $r(1 \leq r<2)$, and think of $\epsilon>0$ as a variable. We construct a homeomorphism $\tau_{1+\epsilon}^{r}: M_{2 m+1, r} \rightarrow M_{2 m+1,1+\epsilon}$ such that $\lim _{\epsilon \rightarrow+0} \tau_{1+\epsilon}^{r}$ exists and defines a homeomorphism. We write this limit as $\tau_{1}^{r}$. Finally define $\tau_{r}^{1}$, which we need to define, as $\left(\tau_{1}^{r}\right)^{-1}$. (Note that $\tau_{1}^{r}$ is a homeomorphism.)

Since constructions of $\tau_{1+\epsilon}^{r}$ are similar for a fixed $r$, we set $r=\frac{3}{2}$ in order to make sure that $r$ is fixed. In order to construct $\tau_{1+\epsilon}^{\frac{3}{2}}: M_{2 m+1, \frac{3}{2}} \rightarrow M_{2 m+1,1+\epsilon}$, we deform the triangle $\left(\beta\left(\frac{3}{2}, \alpha\right), \alpha, \gamma\left(\frac{3}{2}, \alpha\right)\right)$ in the same way as in (A)-(i), i.e., define $h_{1+\epsilon}^{\frac{3}{2}}(\alpha)$ by

$$
h_{1+\epsilon}^{\frac{2}{3}}(\alpha)= \begin{cases}\lambda \psi_{1+\epsilon} & \text { if } \alpha=\lambda \psi_{\frac{3}{2}}(0 \leq \lambda \leq 1), \\ \lambda \psi_{1+\epsilon}+(1-\lambda) \pi & \text { if } \alpha=\lambda \psi_{\frac{3}{2}}+(1-\lambda) \pi(0 \leq \lambda \leq 1) .\end{cases}
$$

(Recall that $s\left(1+\epsilon, \psi_{1+\epsilon}\right)=1$.) Then the deformed triangle is given by

$$
\left(\beta\left(1+\epsilon, h_{1+\epsilon}^{\frac{3}{2}}(\alpha)\right), h_{1+\epsilon}^{\frac{3}{2}}(\alpha), \gamma\left(1+\epsilon, h_{1+\epsilon}^{\frac{3}{2}}(\alpha)\right)\right) .
$$

We note that $\beta\left(1+\epsilon, h_{1+\epsilon}^{\frac{3}{2}}(\alpha)\right)$ is not continuous as $\epsilon \rightarrow+0$ and $\alpha \rightarrow 0$ by Lemma 2.7. In order to make $\tau_{1+\epsilon}^{\frac{3}{2}}$ continuous, we need to deform $\pi_{2 m+1, \frac{3}{2}}^{-1}(\alpha)$ suitably, so that [the deformed triangle] $\dot{+}$ [the deformed $2 m$-gon] is continuous, although [the deformed triangle] itself is not continuous as above.

When $|\alpha|$ is small, we deform $\left(\bar{\theta}_{1}, \bar{\theta}_{3}, \theta_{4}, \ldots, \theta_{2 m+1}\right) \in \pi_{2 m+1, \frac{3}{2}}^{-1}(\alpha)$ by the following idea: As usual, we assume that $\left(\bar{\theta}_{1}, \bar{\theta}_{3}, \theta_{4}, \ldots, \theta_{2 m+1}\right) \in M_{2 m, s\left(\frac{3}{2}, \alpha\right)}$ by $\mu\left(\frac{3}{2}, \alpha\right)$. Write $\rho_{s\left(1+\epsilon, h_{1+\epsilon}^{\frac{3}{2}}, \alpha\right)}^{\left.\frac{3}{2}(\alpha)\right)}\left(\bar{\theta}_{1}, \bar{\theta}_{3}, \theta_{4}, \ldots, \theta_{2 m+1}\right)$ as $\left(\bar{\theta}_{1}^{\prime}, \bar{\theta}_{3}^{\prime}, \theta_{4}^{\prime}, \ldots, \theta_{2 m+1}^{\prime}\right)$. If $\left(\bar{\theta}_{1}, \bar{\theta}_{3}, \theta_{4}, \ldots, \theta_{2 m+1}\right)$ can be deformed to

$$
\left(\bar{\theta}_{1}^{\prime \prime}, \bar{\theta}_{3}^{\prime \prime}, \theta_{4}^{\prime \prime}, \ldots, \theta_{2 m+1}^{\prime \prime}\right) \in M_{2 m, s\left(1+\epsilon, h_{1+\epsilon}^{\frac{3}{2}}(\alpha)\right)}
$$

so that

$$
\begin{aligned}
& \bar{\theta}_{1}^{\prime \prime} \approx \bar{\theta}_{1}^{\prime}-\left\{\gamma\left(\frac{3}{2}, \alpha\right)-\gamma\left(1+\epsilon, h_{1+\epsilon}^{\frac{3}{2}}(\alpha)\right)\right\}, \\
& \bar{\theta}_{3}^{\prime \prime} \approx \bar{\theta}_{3}^{\prime}+\left\{\gamma\left(\frac{3}{2}, \alpha\right)-\gamma\left(1+\epsilon, h_{1+\epsilon}^{\frac{3}{2}}(\alpha)\right)\right\}, \\
& \theta_{4}^{\prime \prime} \approx \theta_{4}^{\prime}, \ldots, \theta_{2 m+1}^{\prime \prime} \approx \theta_{2 m+1}^{\prime}
\end{aligned}
$$


$(\approx$ means approximately the same $)$, then we can define $\tau_{1+\epsilon}^{\frac{3}{2}}\left(\theta_{1}, \alpha, \theta_{3}, \ldots, \theta_{2 m+1}\right)$ by

$$
\begin{aligned}
\tau_{1+\epsilon}^{\frac{3}{2}}\left(\theta_{1}, \alpha, \theta_{3}, \ldots, \theta_{2 m+1}\right)= & \left(\beta\left(1+\epsilon, h_{1+\epsilon}^{\frac{3}{2}}(\alpha)\right), h_{1+\epsilon}^{\frac{3}{2}}(\alpha), \gamma\left(1+\epsilon, h_{1+\epsilon}^{\frac{3}{2}}(\alpha)\right)\right) \\
& \dot{+}\left(\bar{\theta}_{1}^{\prime \prime}, \bar{\theta}_{3}^{\prime \prime}, \theta_{4}^{\prime \prime}, \ldots, \theta_{2 m+1}^{\prime \prime}\right) .
\end{aligned}
$$

In fact, by (3.14) we have

$$
\begin{aligned}
\tau_{1+\epsilon}^{\frac{3}{2}}\left(\theta_{1}, \alpha, \theta_{3}, \ldots, \theta_{2 m+1}\right) \approx & \left(\bar{\theta}_{1}^{\prime}+\beta\left(1+\epsilon, h_{1+\epsilon}^{\frac{3}{2}}(\alpha)\right)+\gamma\left(1+\epsilon, h_{1+\epsilon}^{\frac{3}{2}}(\alpha)\right)-\gamma\left(\frac{3}{2}, \alpha\right),\right. \\
& \left.h_{1+\epsilon}^{\frac{3}{2}}(\alpha), \bar{\theta}_{3}^{\prime}+\gamma\left(\frac{3}{2}, \alpha\right), \theta_{4}^{\prime}, \ldots, \theta_{2 m+1}^{\prime}\right) .
\end{aligned}
$$

We know that

$$
\lim _{\substack{\alpha \rightarrow 0 \\ r \rightarrow 1}} \beta(r, \alpha)+\gamma(r, \alpha)=\pi
$$

in the notation of Lemma 2.7. Hence the term

$$
\beta\left(1+\epsilon, h_{1+\epsilon}^{\frac{3}{2}}(\alpha)\right)+\gamma\left(1+\epsilon, h_{1+\epsilon}^{\frac{3}{2}}(\alpha)\right)
$$

in (3.16) is continuous as $\epsilon \rightarrow+0$ and $\alpha \rightarrow 0$. Thus $\tau_{1+\epsilon}^{\frac{3}{2}}$ is continuous.

For small $|\alpha|$, the deformation of $\left(\bar{\theta}_{1}, \bar{\theta}_{3}, \theta_{4}, \ldots, \theta_{2 m+1}\right)$ to $\left(\bar{\theta}_{1}^{\prime \prime}, \bar{\theta}_{3}^{\prime \prime}, \theta_{4}^{\prime \prime}, \ldots, \theta_{2 m+1}^{\prime \prime}\right)$ is defined as follows.

(a) First get an element $\rho_{0}^{s\left(\frac{3}{2}, \alpha\right)}\left(\bar{\theta}_{1}, \bar{\theta}_{3}, \theta_{4}, \ldots, \theta_{2 m+1}\right) \in M_{2 m, 0}$, and identify $M_{2 m, 0}$ with $S^{1} \times M_{2 m-1,1}$ by $\eta$ (cf. Lemma 2.9.) So we can write the above element as $\left(\sigma,\left(\Theta_{1}, \Theta_{4}, \Theta_{5}, \ldots, \Theta_{2 m+1}\right)\right)$, where $\sigma, \Theta_{1}, \Theta_{4}, \Theta_{5}, \ldots, \Theta_{2 m+1} \in[0,2 \pi]$.

(b) Next form $\left(\sigma+\gamma\left(\frac{3}{2}, \alpha\right)-\gamma\left(1+\epsilon, h_{1+\epsilon}^{\frac{3}{2}}(\alpha)\right),\left(\Theta_{1}, \Theta_{4}, \ldots, \Theta_{2 m+1}\right)\right)$, i.e., rotate $\left(\sigma,\left(\Theta_{1}, \Theta_{4}, \ldots, \Theta_{2 m+1}\right)\right)$ by $\gamma\left(\frac{3}{2}, \alpha\right)-\gamma\left(1+\epsilon, h_{1+\epsilon}^{\frac{3}{2}}(\alpha)\right)$ in the counter-clockwise angle.

(c) Finally get an element

$$
\begin{aligned}
& \rho_{s\left(1+\epsilon, h_{1+\epsilon}^{\frac{3}{2}}(\alpha)\right)}^{0}\left(\sigma+\gamma\left(\frac{3}{2}, \alpha\right)-\gamma\left(1+\epsilon, h_{1+\epsilon}^{\frac{3}{2}}(\alpha)\right),\left(\Theta_{1}, \Theta_{4}, \ldots, \Theta_{2 m+1}\right)\right) \\
& \quad \in M_{2 m, s\left(1+\epsilon, h_{1+\epsilon}^{\frac{3}{2}}(\alpha)\right) .}
\end{aligned}
$$

(By using the definition of $\rho_{r}^{0}$, it is easily checked that the steps (a), (b) and (c) satisfy (3.14).)

Then we finally need to extend this $\tau_{1+\epsilon}^{\frac{3}{2}}$ continuously for any $\alpha$. (As usual we need to deform a singular fiber $\pi_{2 m+1, \frac{3}{2}}^{-1}\left(\psi_{\frac{3}{2}}\right)$ to $\pi_{2 m+1,1+\epsilon}^{-1}\left(\psi_{1+\epsilon}\right)$.)

Thus it is natural to define $\tau_{1+\epsilon}^{\frac{3}{2}}$ in the following manner: Choose a small positive number, say $\frac{1}{100}$, then choose a continuous function $F:[0, \pi] \rightarrow[0,1]$ so that $F(\alpha)=1$ for $0 \leq \alpha \leq \psi_{\frac{3}{2}}-\frac{1}{100}$, and $F(\alpha)=0$ for $\psi_{\frac{3}{2}} \leq \alpha \leq \pi$. Then extend the domain of $F$ to $[0,2 \pi]$ by setting $F(-\alpha)=F(\alpha)$ for $\alpha \in[0, \pi]$.

Take an element $\left(\theta_{1}, \alpha, \theta_{3}, \ldots, \theta_{2 m+1}\right) \in M_{2 m+1, \frac{3}{2}}$.

(1) If $s\left(\frac{3}{2}, \alpha\right)<1$, then write $\rho_{0}^{s\left(\frac{3}{2}, \alpha\right)}\left(\bar{\theta}_{1}, \bar{\theta}_{3}, \theta_{4}, \ldots, \theta_{2 m+1}\right)$ as

$$
\left(\sigma,\left(\Theta_{1}, \Theta_{4}, \ldots, \Theta_{2 m+1}\right)\right) \text {. }
$$


And set

$$
\begin{aligned}
& \tau_{1+\epsilon}^{\frac{3}{2}}\left(\theta_{1}, \alpha, \theta_{3}, \ldots, \theta_{2 m+1}\right)=\left(\beta\left(1+\epsilon, h_{1+\epsilon}^{\frac{3}{2}}(\alpha)\right), h_{1+\epsilon}^{\frac{3}{2}}(\alpha), \gamma\left(1+\epsilon, h_{1+\epsilon}^{\frac{3}{2}}(\alpha)\right)\right) \\
& \left.\dot{+} \rho_{s\left(1+\epsilon, h_{1+\epsilon}^{0}\right.}^{\frac{3}{2}}(\alpha)\right) \\
& \quad\left(\sigma+F(\alpha)\left\{\gamma\left(\frac{3}{2}, \alpha\right)-\gamma\left(1+\epsilon, h_{1+\epsilon}^{\frac{3}{2}}(\alpha)\right)\right\},\left(\Theta_{1}, \Theta_{4}, \ldots, \Theta_{2 m+1}\right)\right) .
\end{aligned}
$$

(2) If $s\left(\frac{3}{2}, \alpha\right)>1$, then set

$$
\begin{aligned}
\tau_{1+\epsilon}^{\frac{3}{2}}\left(\theta_{1}, \alpha, \theta_{3}, \ldots, \theta_{2 m+1}\right)= & \left(\beta\left(1+\epsilon, h_{1+\epsilon}^{\frac{3}{2}}(\alpha)\right), h_{1+\epsilon}^{\frac{3}{2}}(\alpha), \gamma\left(1+\epsilon, h_{1+\epsilon}^{\frac{3}{2}}(\alpha)\right)\right) \\
& \left.\dot{+} \rho_{s\left(1+\epsilon, h_{1+\epsilon}^{2}, \alpha\right)}^{\frac{3}{2}}(\alpha)\right) \\
& \left(\bar{\theta}_{1}, \bar{\theta}_{3}, \theta_{4}, \ldots, \theta_{2 m+1}\right) .
\end{aligned}
$$

(3) If $s\left(\frac{3}{2}, \alpha\right)=1$, then set

$$
\begin{aligned}
\tau_{1+\epsilon}^{\frac{3}{2}}\left(\theta_{1}, \alpha, \theta_{3}, \ldots, \theta_{2 m+1}\right)= & \left(\beta\left(1+\epsilon, h_{1+\epsilon}^{\frac{3}{2}}(\alpha)\right), h_{1+\epsilon}^{\frac{3}{2}}(\alpha), \gamma\left(1+\epsilon, h_{1+\epsilon}^{\frac{3}{2}}(\alpha)\right)\right) \\
& \dot{+}\left(\bar{\theta}_{1}, \bar{\theta}_{3}, \theta_{4}, \ldots, \theta_{2 m+1}\right) .
\end{aligned}
$$

We can easily prove that $\lim _{\epsilon \rightarrow+0} \tau_{1+\epsilon}^{\frac{3}{2}}$ exists and defines a homeomorphism. This completes the construction of $\tau_{r}^{1}(0<r<2)$, and consequently, the proof of Theorem 3.1 .

\section{MOdels FOR $M_{n, k}$}

In this section, we construct a space which has the same homotopy type as $M_{n, k}$, where $k \in \mathbf{Z}$. We prepare some notations.

We consider the following diagram:

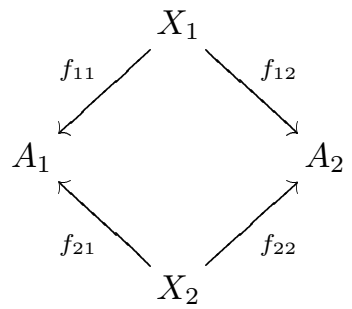

where $X_{1}, X_{2}, A_{1}, A_{2}$ are spaces and $f_{i j}$ are continuous maps. To this diagram, we correspond a space, which is called the homotopy colimit [1], as follows. Homotopy colimit is a quotient space obtained from the topological sum of $X_{1} \times I, X_{2} \times I, A_{1}$ and $A_{2}$ by identifying $\left(x_{i},-1\right) \in X_{i} \times I$ with $f_{i 1}\left(x_{i}\right) \in A_{1}$ and $\left(x_{i}, 1\right) \in X_{i} \times I$ with $f_{i 2}\left(x_{i}\right) \in A_{2}$ for $i=1,2$, where $I=[-1,1]$.

Let us write this homotopy colimit by

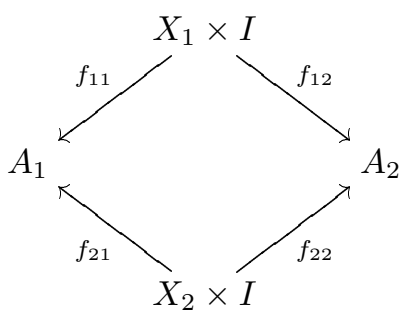


Let $\mathcal{H}$ o be the homotopy category. (For two topological spaces $X$ and $Y, X=Y$ in $\mathcal{H}$ o means that $X$ and $Y$ are homotopy equivalent. And for $f, g: X \rightarrow Y, f=g$ means that $f$ is homotopic to $g$.)

As for the homotopy colimit, the following lemma is well known.

Lemma 4.2. The homotopy colimit is a homotopy functor (from the category of diagrams to the homotopy category). Thus the homotopy colimit depends only on the homotopy equivalences of $X_{i}$ and the homotopy classes of $f_{i j}$.

In the following Definitions 4.3 and $4.3^{\circ}$, we consider special diagrams.

Definition 4.3. We consider the following diagram:

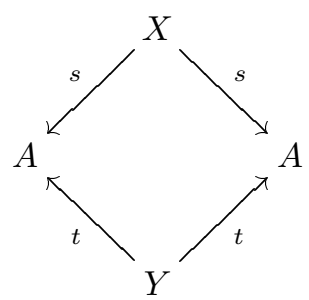

We denote its homotopy colimit by $I(s, t)$. In particular, if $Y=A$ and $t=i d_{A}$ : $A \rightarrow A$, then we denote $I\left(s, i d_{A}\right)$ by $J(s)$.

Definition $4.3^{\circ}$. We consider the following diagram:

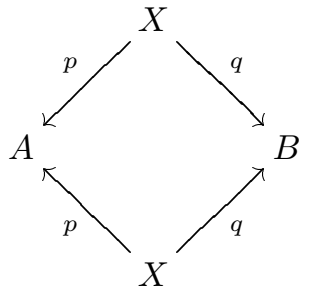

We denote its homotopy colimit by $I I(p, q)$. In particular, if $X=B$ and $q=i d_{B}$ : $B \rightarrow B$, then we denote $I I\left(p, \operatorname{id}_{B}\right)$ by $J^{\prime}(p)$.

Lemma 4.4. In $\mathcal{H}$ o, we have $J(s)=J^{\prime}(s)$ for $s: X \rightarrow A$.

Proof. The definitions of $J(s)$ and $J^{\prime}(s)$ are as follows:
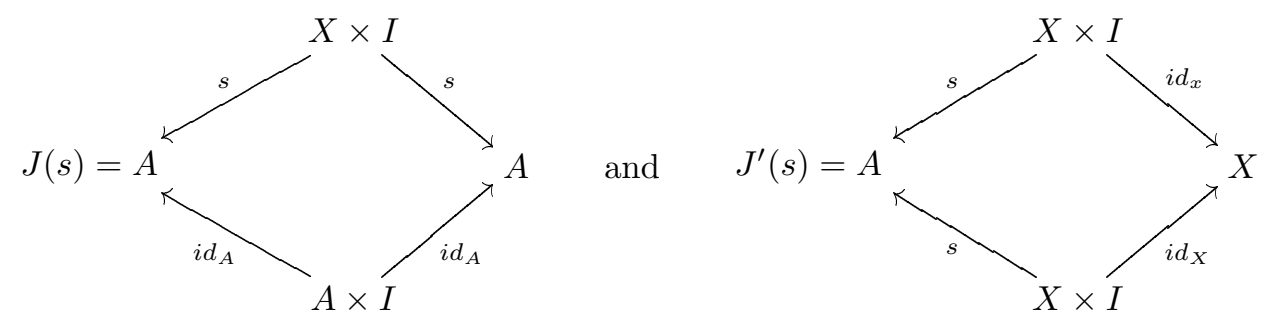

We define a map $J(s) \rightarrow J^{\prime}(s)$. Let $c: I \rightarrow I$ be the scalar change defined by $c(x)=2 x+1,-1 \leq x \leq 0$ and $c(x)=-2 x+1,0 \leq x \leq 1$. Then the map is defined by sending $A \times I$ in $J(s)$ to $A$ in $J^{\prime}(s)$ by the projection to the first factor, and then sending $X \times I$ in $J(s)$ to $X \times I \cup X \times I$ in $J^{\prime}(s)$ via $i d_{X} \times c$. We see that the map induces a homotopy equivalence. 
We will define the contraction maps which play the essential role to attain our purpose.

Definition 4.5. (i) To a diagram in Definition 4.3, we define its lower contraction as follows:
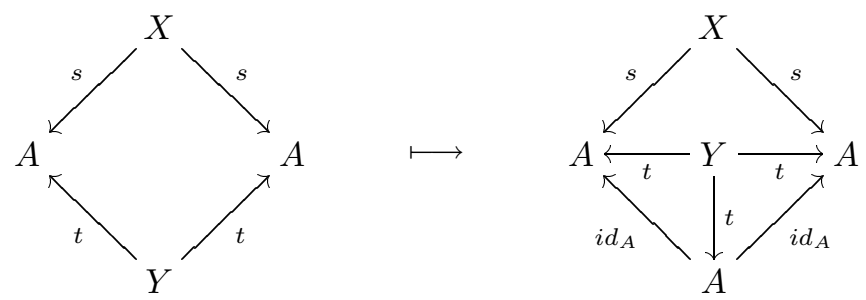

This morphism induces a map between homotopy colimits $\gamma_{1}(s): I(s, t) \rightarrow$ $J(s)$.

(ii) Similarly we can define the upper contraction map $\gamma_{2}(t): I(s, t) \rightarrow J(t)$.

(iii) If we consider (ii) to the case when $Y=A$ and $t=i d_{A}: A \rightarrow A$, then we have a map $\gamma_{2}\left(i d_{A}\right): I\left(s, i d_{A}\right) \rightarrow J\left(i d_{A}\right)$. Since $I\left(s, i d_{A}\right)=J(s)$ and $J\left(i d_{A}\right) \simeq S^{1} \times A$, we can write this map as $\gamma_{2}: J(s) \rightarrow S^{1} \times A$.

Definition $4.5^{\circ}$. (i) For a diagram

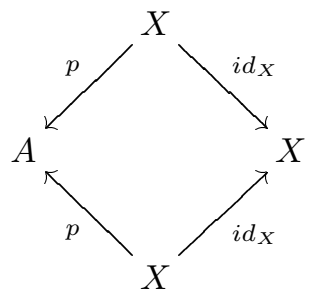

and a map $q: X \rightarrow B$, we define its right contraction as follows:
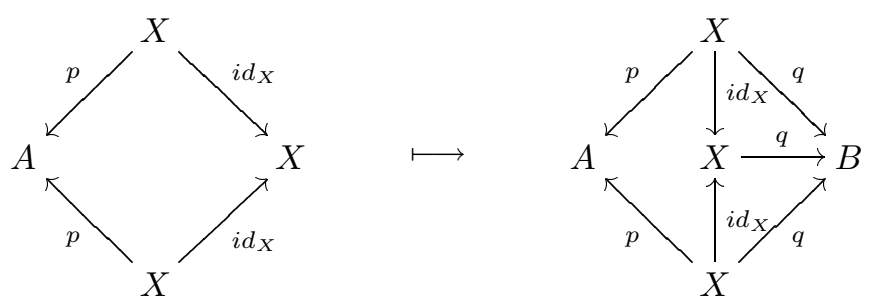

This morphism induces a map between homotopy colimits $\lambda_{1}(q): J^{\prime}(p) \rightarrow$ $I I(p, q)$.

(ii) Similarly, for a diagram

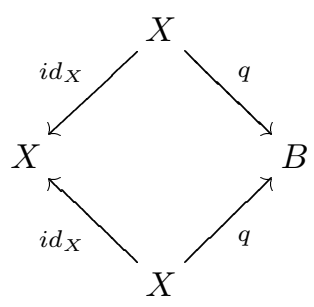


and a map $p: X \rightarrow A$, we can define the left contraction map $\lambda_{2}(p): J^{\prime}(q) \rightarrow$ $I I(p, q)$.

(iii) If we consider (ii) in the case when $B=X$ and $q=i d_{X}: X \rightarrow X$, then we have a map $\lambda_{2}(p): S^{1} \times X \rightarrow J^{\prime}(p)$, which is induced from the following map:
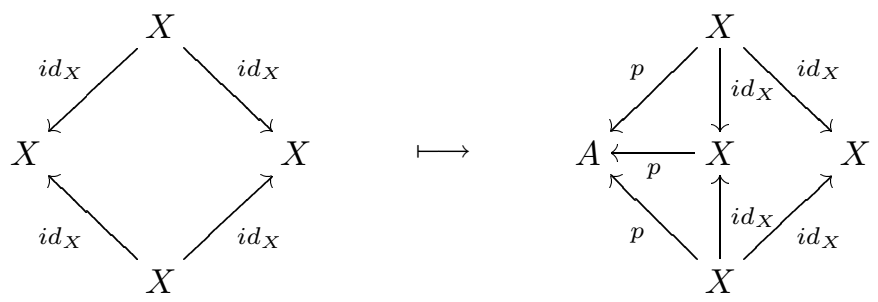

Theorem 4.6. In $\mathcal{H o}$, we have

$$
M_{n, k}= \begin{cases}I\left(f_{n-1, k+1}^{-}, f_{n-1, k-1}^{+}\right) & \text {if } n-k \text { is even and } k \geq 1, \\ I I\left(f_{n-1, k}^{+}, f_{n-1, k}^{-}\right) & \text {if } n-k \text { is odd and } k \geq 1 .\end{cases}
$$

We recall that $f_{n-1, k}^{ \pm}: M_{n-1, k} \rightarrow M_{n-1, k \pm 1}$ are defined in Theorem 3.1.

Proof. For $2 \leq k \leq n-3$, this theorem is the direct translation of Theorem 3.1. For $k \geq n-2$, this theorem is still valid if we remember that the empty set has the unique map $\emptyset$ into any space.

So there remains the case $M_{n, 1}$. We only consider $M_{2 m, 1}$. We define a map (-1) : $M_{2 m-1,0} \rightarrow M_{2 m-1,0}$ as taking $\left(u_{1}, u_{2}, \ldots, u_{2 m-1}\right)$ to $\left(-u_{1},-u_{2}, \ldots,-u_{2 m-1}\right)$, where we note that $u_{1}=u_{2}=0$ in this case. By Theorem 3.1 (see the construction of $\left.\tau_{1+\epsilon}^{\frac{3}{2}}\right), M_{2 m, 1}$ is homeomorphic to

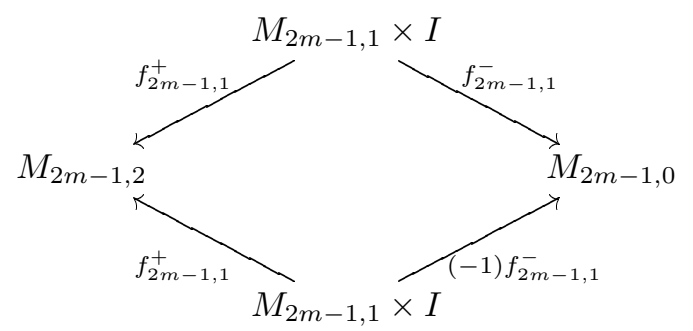

Hence in order to complete the proof, it is enough to show that $(-1)$ is homotopic to the identity by Lemma 4.2. If we define a homotopy $\overline{f_{t}}: S^{1} \times M_{2 m-2,1} \rightarrow$ $S^{1} \times M_{2 m-2,1}$ by a rotation $\overline{f_{t}}(\alpha, u)=\left(e^{i \pi t} \alpha, u\right)$ with $0 \leq t \leq 1, \alpha \in S^{1}$ and $u \in M_{2 m-2,1}$, then the induced homotopy $f_{t}=\eta^{-1} \cdot \overline{f_{t}} \cdot \eta: M_{2 m-1,0} \rightarrow M_{2 m-1,0}$ $\left(\eta: M_{2 m-1,0} \stackrel{\simeq}{\longrightarrow} S^{1} \times M_{2 m-2,1}\right.$ is defined in Lemma 2.9$)$ is the desired homotopy.

We can apply the same argument to $M_{2 m+1,1}$.

Theorem 4.7. We have the following commutative diagrams in $\mathcal{H o .}$

(A) (i) For $n-k \equiv 0 \bmod 2$ and $k \geq 1$,

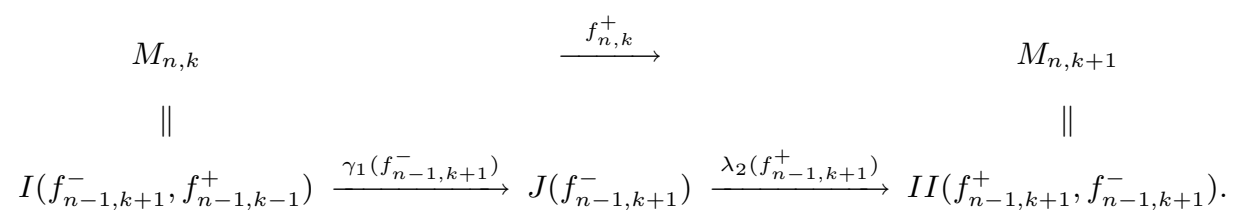


(ii) For $n-k \equiv 0 \bmod 2$ and $k \geq 2$,

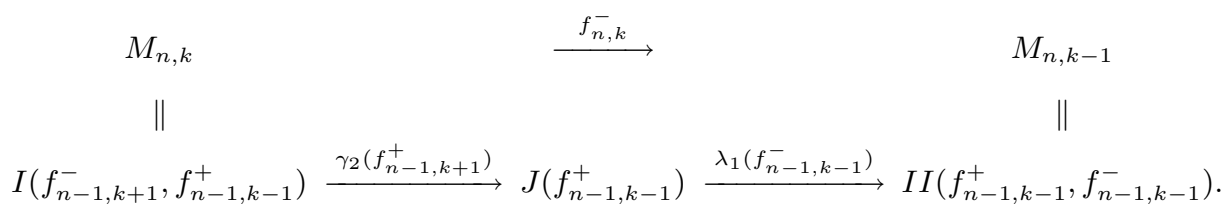

(B) (iii)

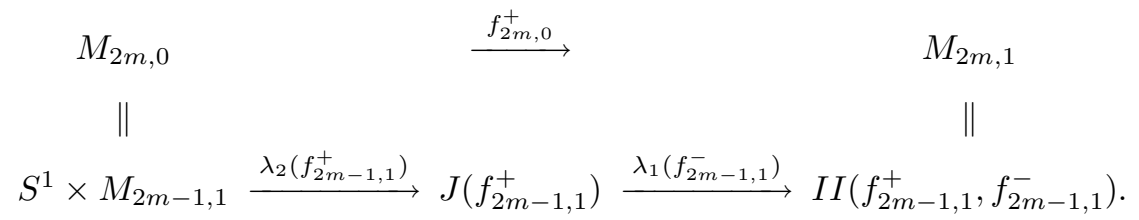

(iv)

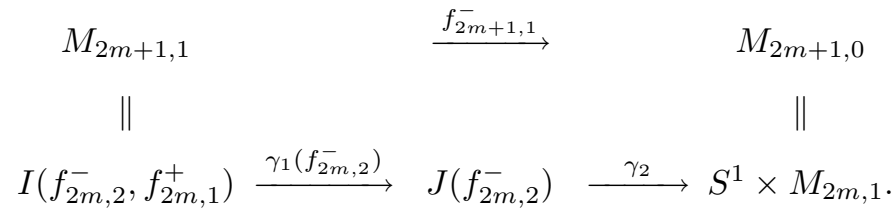

Proof. We will prove A (i), as the other cases are handled in a similar way. Recall that $I\left(f_{n-1, k+1}^{-}, f_{n-1, k-1}^{+}\right)$and $I I\left(f_{n-1, k+1}^{+}, f_{n-1, k+1}^{-}\right)$are defined by the following colimits:

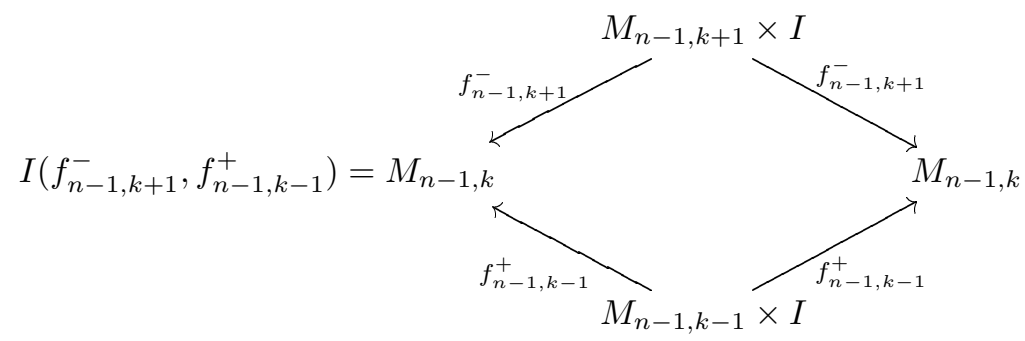

and

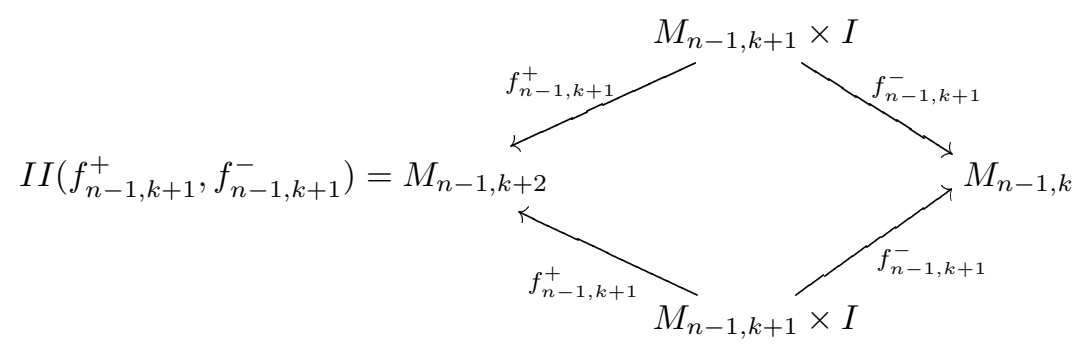

where we recall that two copies of $M_{n-1, k}$ in $I\left(f_{n-1, k+1}^{-}, f_{n-1, k-1}^{+}\right)$correspond to the singular fibers of the projection $\pi_{n, k}: M_{n, k} \rightarrow S^{1}$ (see $\S 2$ ).

By the construction of $f_{n, k}^{+}: M_{n, k} \rightarrow M_{n, k+1}$, this map is characterized as follows.

(i) For $(u, t) \in M_{n-1, k-1} \times I$, we have

$$
f_{n, k}^{+}(u, t)=f_{n-1, k-1}^{+}(u) \in I I\left(f_{n-1, k+1}^{+}, f_{n-1, k+1}^{-}\right) .
$$


(ii) $f_{n, k}^{+}$maps $M_{n-1, k+1} \times I$ to $M_{n-1, k+1} \times I \cup M_{n-1, k+1} \times I$ by a scalar change of $I$ (see $c$ in the proof of Lemma 4.4).

Next we look at $\lambda_{2}\left(f_{n-1, k+1}^{-}\right) \gamma_{1}\left(f_{n-1, k+1}^{-}\right)$.

(iii) Recall that $J\left(f_{n-1, k+1}^{-}\right)$is defined by the following colimit:

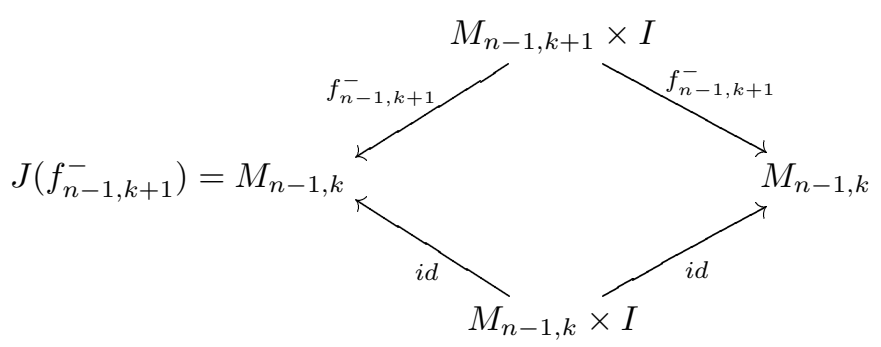

It is easy to see that $J\left(f_{n-1, k+1}^{-}\right)$is homotopy equivalent to a quotient space obtained from the topological sum of $M_{n-1, k+1} \times I$ and $M_{n-1, k}$ by identifying $(u, 0) \in M_{n-1, k+1} \times I$ with $f_{n-1, k+1}^{-}(u) \in M_{n-1, k}$, and $(u, 1) \in M_{n-1, k+1} \times I$ with $f_{n-1, k+1}^{-}(u) \in M_{n-1, k}$. Hence by $\gamma_{1}\left(f_{n-1, k+1}^{-}\right),(u, t) \in M_{n-1, k-1} \times I$ is mapped to $f_{n-1, k-1}^{+}(u) \in M_{n-1, k}$.

(iv) By $\lambda_{2}\left(f_{n-1, k+1}^{+}\right), f_{n-1, k-1}^{+}(u) \in M_{n-1, k}$ (see (iii)) is mapped to itself in $I I\left(f_{n-1, k+1}^{+}, f_{n-1, k+1}^{-}\right)$.

Now by (i)-(ii) and (iii)-(iv), we see that $f_{n, k}^{+}$and $\lambda_{2}\left(f_{n-1, k+1}^{-}\right) \gamma_{1}\left(f_{n-1, k+1}^{-}\right)$are homotopic.

\section{Auxiliary theorems}

We prepare some theorems which are useful to clarify the proof of Theorems B and $\mathrm{C}$. We begin by proving two lemmas.

Lemma 5.1. We consider the diagram

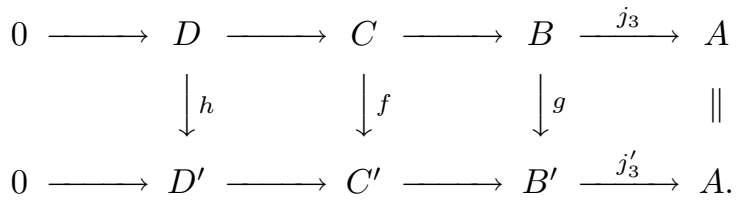

Here the upper and lower sequences are exact and $h$ is surjective. Then we have the following isomorphism (i) and an exact sequence (ii).

(i) Coker $f \cong\left(\operatorname{Ker} j_{3}^{\prime}+\operatorname{Im} g\right) / \operatorname{Im} g$.

(ii) $0 \longrightarrow \operatorname{Ker} h \longrightarrow \operatorname{Ker} f \longrightarrow \operatorname{Ker} g \longrightarrow 0$.

Proof. We have the diagram of two exact sequences

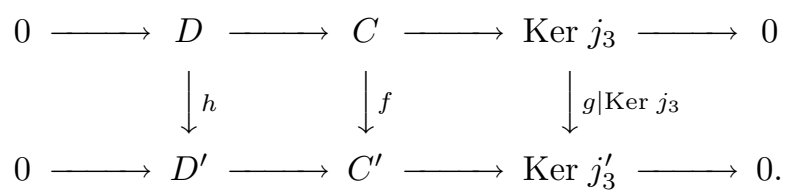

Hence we get the long exact sequence

$$
\begin{aligned}
& 0 \longrightarrow \operatorname{Ker} h \longrightarrow \operatorname{Ker} f \longrightarrow \operatorname{Ker}\left(g \mid \operatorname{Ker} j_{3}\right) \longrightarrow \text { Coker } h \longrightarrow \text { Coker } f \\
& \quad \longrightarrow \text { Coker }\left(g \mid \text { Ker } j_{3}\right) \longrightarrow 0 .
\end{aligned}
$$


Since $h$ is surjective, we have isomorphisms

$$
0 \longrightarrow \text { Coker } f \longrightarrow \text { Coker }\left(g \mid \text { Ker } j_{3}\right) \longrightarrow 0
$$

and

$$
0 \longrightarrow \operatorname{Ker} h \longrightarrow \operatorname{Ker} f \longrightarrow \operatorname{Ker}\left(g \mid \operatorname{Ker} j_{3}\right) \longrightarrow 0 \text {. }
$$

From the definition, we have

$$
\text { Coker }\left(g \mid \operatorname{Ker} j_{3}\right)=\operatorname{Ker} j_{3}^{\prime} /\left(\operatorname{Im} g \cap \operatorname{Ker} j_{3}^{\prime}\right) \cong\left(\operatorname{Ker} j_{3}^{\prime}+\operatorname{Im} g\right) / \operatorname{Im} g \text {. }
$$

Since $j_{3}=j_{3}^{\prime} g$, we see that Ker $g \subset \operatorname{Ker} j_{3}$. Hence we get

$$
\operatorname{Ker}\left(g \mid \operatorname{Ker} j_{3}\right)=\operatorname{Ker} g \cap \operatorname{Ker} j_{3}=\operatorname{Ker} g \text {. }
$$

Now the lemma follows from (5.2)

Dually we have

Lemma 5.3. We consider the diagram

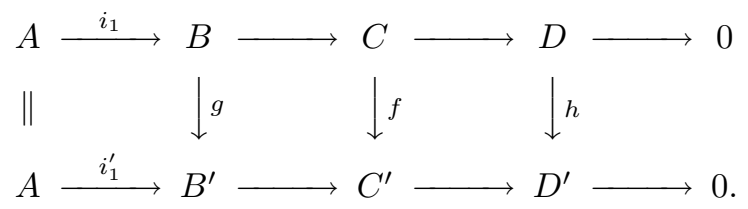

Here the upper and lower sequences are exact and $h$ is injective. Then we have the following isomorphism (i) and an exact sequence (ii).

(i) $\operatorname{Ker} f \cong \operatorname{Ker} g /\left(\operatorname{Ker} g \cap \operatorname{Im} i_{1}\right)$.

(ii) $0 \longrightarrow$ Coker $g \longrightarrow$ Coker $f \longrightarrow$ Coker $h \longrightarrow 0$.

Proof. We have the diagram of two exact sequences

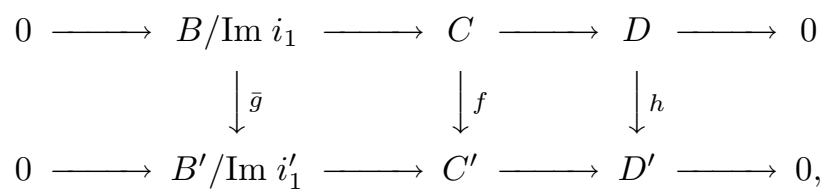

where $\bar{g}$ is the induced map of $g$. Hence we get the long exact sequence

$$
0 \longrightarrow \text { Ker } \bar{g} \longrightarrow \text { Ker } f \longrightarrow \text { Ker } h \longrightarrow \text { Coker } \bar{g} \longrightarrow \text { Coker } f \longrightarrow \text { Coker } h \longrightarrow 0 \text {. }
$$

Since $h$ is injective, we get

$$
0 \longrightarrow \operatorname{Ker} \bar{g} \longrightarrow \operatorname{Ker} f \longrightarrow 0
$$

and

$$
0 \longrightarrow \text { Coker } \bar{g} \longrightarrow \text { Coker } f \longrightarrow \text { Coker } h \longrightarrow 0 .
$$

From the definition, we have

$$
\text { Ker } f \cong \operatorname{Ker} \bar{g}=\left(\operatorname{Ker} g+\operatorname{Im} i_{1}\right) / \operatorname{Im} i_{1} \cong \operatorname{Ker} g /\left(\operatorname{Ker} g \cap \operatorname{Im} i_{1}\right)
$$

and

$$
\text { Coker } \bar{g}=\left(B^{\prime} / \operatorname{Im} i_{1}^{\prime}\right) /\left(\left(\operatorname{Im} g+\operatorname{Im} i_{1}^{\prime}\right) / \operatorname{Im} i_{1}^{\prime}\right) \cong B^{\prime} /\left(\operatorname{Im} g+\operatorname{Im} i_{1}^{\prime}\right) \text {. }
$$

Noting that $i_{1}^{\prime}=g i_{1}$, we see that $\operatorname{Im} i_{1}^{\prime} \subset \operatorname{Im} g$. It means that

Coker $\bar{g} \cong B^{\prime} / \operatorname{Im} g=$ Coker $g$.

Now the lemma follows from (5.4). 
Notation 5.5. We collect notations here. Let $K$ be any field. Then we denote the homology with $K$-coefficients by $H_{*}(X)$. We assume that $H_{n}(X)=0$ for $X=\emptyset$ or $n<0$. Furthermore we assume that the map $\emptyset$ from the empty set to any space induces $\emptyset_{*}=0$ on homology groups.

Theorem 5.6. For $s: X \rightarrow A$ and $t: Y \rightarrow A$, we assume that $\operatorname{Im}\left(s_{*}\right) \subset \operatorname{Im}\left(t_{*}\right)$. Then the following (i) and (ii) hold.

(i) We have the following isomorphism:

$$
H_{j}(I(s, t)) \cong H_{j}(A) \oplus \operatorname{Coker}_{j}\left(t_{*}\right) \oplus H_{j-1}(X) \oplus \operatorname{Ker}_{j-1}\left(t_{*}\right) .
$$

In particular, we have

$$
H_{j}(J(t)) \cong H_{j}(A) \oplus H_{j-1}(Y)
$$

As for $I(s, t)$ and $J(t)$, see $\S 4$.

(ii) We have the following exact sequences:

$$
0 \longrightarrow \operatorname{Coker}_{j}\left(t_{*}\right) \oplus \operatorname{Ker}_{j-1}\left(t_{*}\right) \longrightarrow H_{j}(I(s, t)) \stackrel{\gamma_{1}(s)_{*}}{\longrightarrow} H_{j}(J(s)) \longrightarrow 0
$$

and

$$
\begin{aligned}
0 \longrightarrow \operatorname{Coker}_{j}\left(t_{*}\right) \oplus \operatorname{Ker}_{j-1}\left(s_{*}\right) \longrightarrow H_{j}(I(s, t)) \stackrel{\gamma_{2}(t)_{*}}{\longrightarrow} H_{j}(J(t)) \\
\quad \longrightarrow \operatorname{Im}_{j-1}\left(t_{*}\right) / \operatorname{Im}_{j-1}\left(s_{*}\right) \longrightarrow 0 .
\end{aligned}
$$

Proof. (i) From the Mayer-Vietoris sequence for $I(s, t)$, we have

$$
\begin{aligned}
\cdots & \longrightarrow H_{j}(X) \oplus H_{j}(Y) \stackrel{\mathcal{M}_{j}}{\longrightarrow} H_{j}(A) \oplus H_{j}(A) \stackrel{(\iota+)_{*}+\left(\iota_{-}\right)_{*}}{\longrightarrow} H_{j}(I(s, t)) \\
& \stackrel{\partial_{j}}{\longrightarrow} H_{j-1}(X) \oplus H_{j-1}(Y) \stackrel{\mathcal{M}_{j-1}}{\longrightarrow} \cdots,
\end{aligned}
$$

where $\iota_{ \pm}: A \rightarrow I(s, t)$ are the two inclusions and $\mathcal{M}_{j}$ is a map defined by

$$
(x, y) \mapsto\left(s_{*} x-t_{*} y, s_{*} x-t_{*} y\right)
$$

for $x \in H_{j}(X)$ and $y \in H_{j}(Y)$.

It is immediate that $\operatorname{Ker} \mathcal{M}_{j-1} \cong H_{j-1}(X) \oplus \operatorname{Ker}_{j-1}\left(t_{*}\right)$. More explicitly, let $\tau_{j-1}: H_{j-1}(X) \rightarrow H_{j-1}(Y)$ be a map satisfying $t_{*} \tau_{j-1}(x)=s_{*}(x)$. Then Ker $\mathcal{M}_{j-1} \cong \tilde{\Delta} H_{j-1}(X) \oplus \operatorname{Ker}_{j-1}\left(t_{*}\right)$, where we set $\tilde{\Delta}(x)$ equal to $\left(x, \tau_{j-1}(x)\right)$ for $x \in H_{j-1}(X)$.

Let $\Delta: A \rightarrow A \times A$ be the diagonal map. Then we see that $\operatorname{Im} \mathcal{M}_{j}=\Delta \operatorname{Im}_{j}\left(t_{*}\right)$. Furthermore if we write a section of the projection $H_{j}(A) \rightarrow \operatorname{Coker}_{j}\left(t_{*}\right)$ as $\tilde{\sigma}_{j}$, then we have the following decomposition as a vector space:

$$
H_{j}(A) \oplus H_{j}(A) \cong H_{j}(A) \oplus \Delta \operatorname{Im}_{j}\left(t_{*}\right) \oplus \tilde{\sigma}_{j} \operatorname{Coker}_{j}\left(t_{*}\right),
$$

where the first factor of the left-hand side corresponds to the first factor of the right-hand side. Hence we have

$$
\text { Coker } \mathcal{M}_{j} \cong H_{j}(A) \oplus \operatorname{Coker}_{j}\left(t_{*}\right) .
$$

Now we have the exact sequence

$$
0 \longrightarrow H_{j}(A) \oplus \operatorname{Coker}_{j}\left(t_{*}\right) \longrightarrow H_{j}(I(s, t)) \longrightarrow \operatorname{Ker}_{j-1}\left(t_{*}\right) \oplus H_{j-1}(X) \longrightarrow 0 .
$$

This completes the proof of (i). 
(ii) We will give a proof of the second exact sequence. We recall that $J(t)$ is defined by

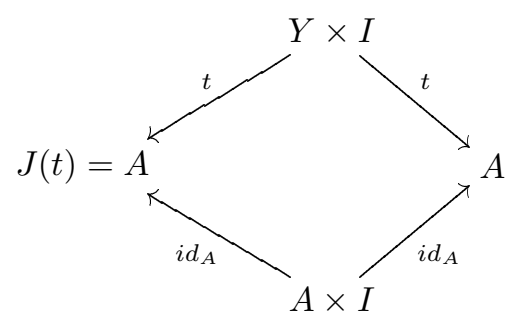

Comparing the Mayer-Vietoris sequences for $I(s, t)$ and $J(t)$, we obtain

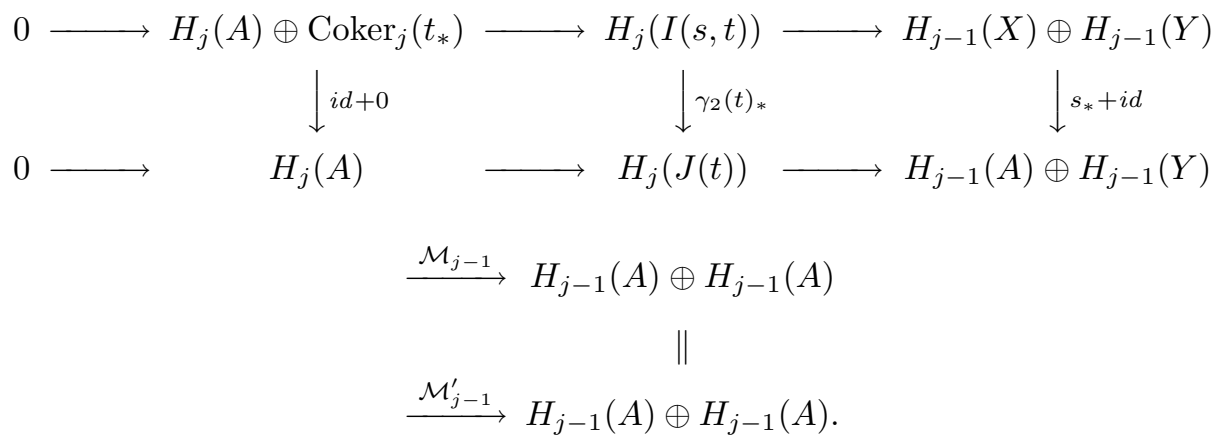

Applying Lemma 5.1, we see that

$$
\operatorname{Coker}_{j}\left(\gamma_{2}(t)_{*}\right) \cong\left(\operatorname{Ker} \mathcal{M}_{j-1}^{\prime}+\operatorname{Im}_{j-1}\left(s_{*}+i d\right)\right) / \operatorname{Im}_{j-1}\left(s_{*}+i d\right)
$$

and

$$
0 \longrightarrow \operatorname{Ker}_{j}(i d+0) \longrightarrow \operatorname{Ker}_{j}\left(\gamma_{2}(t)_{*}\right) \longrightarrow \operatorname{Ker}_{j-1}\left(s_{*}+i d\right) \longrightarrow 0 .
$$

It is easily seen that $\operatorname{Ker} \mathcal{M}_{j-1}^{\prime}=\left\{\left(t_{*} y, y\right): y \in H_{j-1}(Y)\right\} \cong H_{j-1}(Y)$. Since $\operatorname{Im}\left(s_{*}\right) \subset \operatorname{Im}\left(t_{*}\right)$, we see that $\operatorname{Ker} \mathcal{M}_{j-1}^{\prime}+\operatorname{Im}_{j-1}\left(s_{*}+i d\right)=\operatorname{Im}_{j-1}\left(t_{*}\right) \oplus H_{j-1}(Y)$. So we have $\operatorname{Coker}_{j}\left(\gamma_{2}(t)_{*}\right) \cong \operatorname{Im}_{j-1}\left(t_{*}\right) / \operatorname{Im}_{j-1}\left(s_{*}\right)$.

It is immediate that $\operatorname{Ker}_{j}(i d+0)=\operatorname{Coker}_{j}\left(t_{*}\right)$ and $\operatorname{Ker}_{j-1}\left(s_{*}+i d\right)=$ $\operatorname{Ker}_{j-1}\left(s_{*}\right)$. Thus we have $\operatorname{Ker}_{j}\left(\gamma_{2}(t)_{*}\right) \cong \operatorname{Coker}_{j}\left(t_{*}\right) \oplus \operatorname{Ker}_{j-1}\left(s_{*}\right)$.

Now it is easy to prove (ii).

The following theorem is the dual of Theorem 5.6.

Theorem 5.6 ${ }^{\circ}$. For $p: X \rightarrow A$ and $q: X \rightarrow B$, we assume that $\operatorname{Ker}\left(q_{*}\right) \subset$ $\operatorname{Ker}\left(p_{*}\right)$. Then the following (i) and (ii) hold.

(i) We have the following isomorphism:

$$
H_{j}(I I(p, q)) \cong H_{j}(A) \oplus \operatorname{Coker}_{j}\left(q_{*}\right) \oplus H_{j-1}(X) \oplus \operatorname{Ker}_{j-1}\left(q_{*}\right) .
$$

In particular, we have

$$
H_{j}\left(J^{\prime}(p)\right) \cong H_{j}(A) \oplus H_{j-1}(X) .
$$

As for $I I(p, q)$ and $J^{\prime}(p)$, see $\S 4$. 
(ii) We have the following exact sequences:

$$
\begin{aligned}
& \qquad \begin{aligned}
0 & H_{j}\left(J^{\prime}(p)\right) \stackrel{\lambda_{1}(q)}{\longrightarrow} H_{j}(I I(p, q)) \longrightarrow \operatorname{Coker}_{j}\left(q_{*}\right) \oplus \operatorname{Ker}_{j-1}\left(q_{*}\right) \longrightarrow 0 \\
\text { and } & \\
& \longrightarrow \operatorname{Ker}_{j}\left(p_{*}\right) / \operatorname{Ker}_{j}\left(q_{*}\right) \longrightarrow H_{j}\left(J^{\prime}(q)\right) \stackrel{\lambda_{2}(p)_{*}}{\longrightarrow} H_{j}(I I(p, q)) \\
& \longrightarrow \operatorname{Coker}_{j}\left(p_{*}\right) \oplus \operatorname{Ker}_{j-1}\left(q_{*}\right) \longrightarrow 0 .
\end{aligned}
\end{aligned}
$$

Proof. (i) From the Mayer-Vietoris sequence for $I I(p, q)$, we have

$$
\begin{aligned}
\cdots & \longrightarrow H_{j}(X) \oplus H_{j}(X) \stackrel{\mathcal{M}_{j}}{\longrightarrow} H_{j}(A) \oplus H_{j}(B) \stackrel{\left(\iota_{+}\right)_{*}+\left(\iota_{-}\right)_{*}}{\longrightarrow} H_{j}(I I(p, q)) \\
& \stackrel{\partial_{j}}{\longrightarrow} H_{j-1}(X) \oplus H_{j-1}(X) \longrightarrow \cdots,
\end{aligned}
$$

where $\iota_{+}: A \rightarrow I I(p, q)$ and $\iota_{-}: B \rightarrow I I(p, q)$ are the inclusions and $\mathcal{M}_{j}$ is a map defined by

$$
(x, y) \mapsto\left(p_{*} x-p_{*} y, q_{*} x-q_{*} y\right)
$$

for $(x, y) \in H_{j}(X) \oplus H_{j}(X)$.

Since $\operatorname{Ker}\left(q_{*}\right) \subset \operatorname{Ker}\left(p_{*}\right)$, we have $\operatorname{Im} \partial_{j} \cong \operatorname{Ker}_{j-1}\left(q_{*}\right) \oplus \Delta H_{j-1}(X)$, where $\operatorname{Ker}_{j-1}\left(q_{*}\right)$ is a subspace of the first factor of $H_{j-1}(X) \oplus H_{j-1}(X)$ and $\Delta$ : $H_{j-1}(X) \rightarrow H_{j-1}(X) \oplus H_{j-1}(X)$ is the diagonal map. So we see that

Coker $\mathcal{M}_{j} \cong\left(H_{j}(A) \oplus H_{j}(B)\right) / \mathcal{M}_{j}\left(\left(H_{j}(X) \oplus H_{j}(X)\right) /\left(\operatorname{Ker}_{j}\left(q_{*}\right) \oplus \Delta H_{j}(X)\right)\right)$.

Using $H_{j}(X) \oplus H_{j}(X) \cong H_{j}(X) \oplus \Delta H_{j}(X)$, we have

$$
\left(H_{j}(X) \oplus H_{j}(X)\right) /\left(\operatorname{Ker}_{j}\left(q_{*}\right) \oplus \Delta H_{j}(X)\right) \cong H_{j}(X) / \operatorname{Ker}_{j}\left(q_{*}\right) \cong \operatorname{Im}_{j}\left(q_{*}\right) .
$$

Hence we get

$$
\text { Coker } \mathcal{M}_{j} \cong H_{j}(A) \oplus\left(H_{j}(B) / \operatorname{Im}_{j}\left(q_{*}\right)\right) \cong H_{j}(A) \oplus \operatorname{Coker}_{j}\left(q_{*}\right)
$$

Now we have the following exact sequence:

$$
0 \longrightarrow H_{j}(A) \oplus \operatorname{Coker}_{j}\left(q_{*}\right) \longrightarrow H_{j}(I I(p, q)) \longrightarrow H_{j-1}(X) \oplus \operatorname{Ker}_{j-1}\left(q_{*}\right) \longrightarrow 0 \text {. }
$$

This completes the proof of (i).

(ii) We will give a proof of the second exact sequence. Comparing the MayerVietoris sequences for $J^{\prime}(q)$ and $I I(p, q)$, we obtain

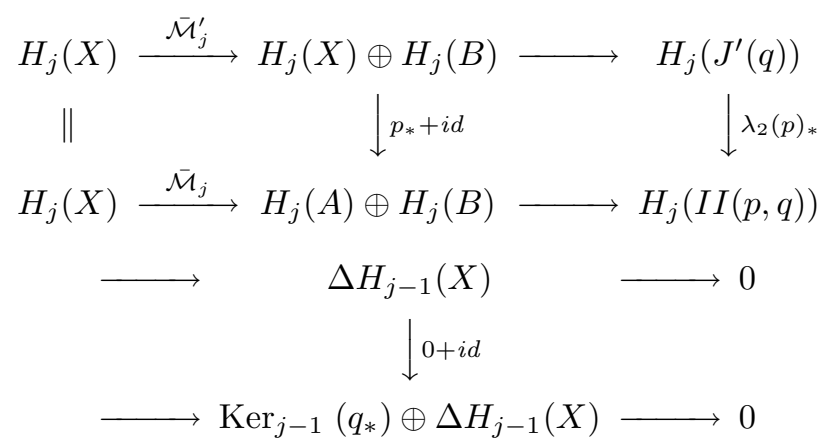

where $\overline{\mathcal{M}}_{j}^{\prime}(\alpha)=\left(\alpha, q_{*} \alpha\right)$ and $\overline{\mathcal{M}}_{j}(\beta)=\left(p_{*} \alpha, q_{*} \beta\right)$ for $\alpha, \beta \in H_{j}(X)$.

Applying Lemma 5.3, we have an isomorphism

$$
\operatorname{Ker}_{j}\left(\lambda_{2}(p)_{*}\right) \cong \operatorname{Ker}_{j}\left(p_{*}+i d\right) /\left(\operatorname{Ker}_{j}\left(p_{*}+i d\right) \cap \operatorname{Im} \overline{\mathcal{M}}_{j}^{\prime}\right)
$$


and an exact sequence

$$
0 \longrightarrow \operatorname{Coker}_{j}\left(p_{*}+i d\right) \longrightarrow \operatorname{Coker}_{j}\left(\lambda_{2}(q)_{*}\right) \longrightarrow \operatorname{Coker}_{j}(0+i d) \longrightarrow 0 .
$$

We see that $\left(\alpha, q_{*} \alpha\right) \in \operatorname{Ker}_{j}\left(p_{*}+i d\right) \cap \operatorname{Im} \overline{\mathcal{M}}_{j}^{\prime}$ if and only if $p_{*} \alpha=0, q_{*} \alpha=0$. From the assumption $\operatorname{Ker}\left(q_{*}\right) \subset \operatorname{Ker}\left(p_{*}\right)$, it follows that $\operatorname{Ker}_{j}\left(p_{*}+i d\right) \cap \operatorname{Im} \overline{\mathcal{M}}_{j}^{\prime} \cong$ $\operatorname{Ker}_{j}\left(q_{*}\right)$.

On the other hand, $\operatorname{Coker}_{j}(0+i d) \cong \operatorname{Ker}_{j-1}\left(q_{*}\right)$.

Now (ii) follows from (5.7).

Theorem 5.8. We have the following two exact sequences for $s: X \rightarrow A$ :

$$
0 \rightarrow \operatorname{Ker}_{j-1}\left(s_{*}\right) \rightarrow H_{j}(J(s)) \stackrel{\left(\gamma_{2}\right)_{*}}{\rightarrow} H_{j}\left(S^{1} \times A\right) \rightarrow \operatorname{Coker}_{j-1}\left(s_{*}\right) \rightarrow 0
$$

and

$$
0 \rightarrow \operatorname{Ker}_{j}\left(s_{*}\right) \rightarrow H_{j}\left(S^{1} \times X\right) \stackrel{\lambda_{2}(s)}{\longrightarrow} H_{j}\left(J^{\prime}(s)\right) \rightarrow \operatorname{Coker}_{j}\left(s_{*}\right) \rightarrow 0 .
$$

Proof. To prove the first exact sequence, we apply the second exact sequence of Theorem 5.6 (ii) for $t=i d_{X}$. Then we can prove the assertion easily.

The second one is proved symmetrically by using Theorem $5.6^{\circ}$.

\section{Homology of $M_{n, k}$}

As in $\S 5$, we abbreviate the homology with $K$-coefficients as $H_{*}(X)$, where $K$ is a field. We recall that $M_{n, n-1}=\{1$-point $\}$ and $M_{n, k}=\emptyset$ for $k \geq n$.

We think of the following assertions.

$A_{n}:\left(f_{n, k}^{+}\right)_{*}: H_{*}\left(M_{n, k}\right) \rightarrow H_{*}\left(M_{n, k+1}\right)$ is surjective if $n-k$ is even and $k \geq 1$.

$B_{n}:\left(f_{n, k}^{-}\right)_{*}: H_{*}\left(M_{n, k}\right) \rightarrow H_{*}\left(M_{n, k-1}\right)$ is injective if $n-k$ is even and $k \geq 2$.

$C_{n}: \operatorname{Im}\left(\left(f_{n, 2}^{-}\right)_{*}\right) \subset \operatorname{Im}\left(\left(f_{n, 0}^{+}\right)_{*}\right)$ if $n$ is even.

$C_{n}^{\circ}: \operatorname{Ker}\left(\left(f_{n, 1}^{-}\right)_{*}\right) \subset \operatorname{Ker}\left(\left(f_{n, 1}^{+}\right)_{*}\right)$ if $n$ is odd.

These are the crucial parts to determine the homology of $M_{n, k}$. In the following, we prove $A_{n}, B_{n}, C_{n}$ and $C_{n}^{\circ}$ for $n \geq 2$ by induction on $n$.

For the first step of the induction, we can easily check the assertions for $n=2$ (see (6.6)). Hence in order to complete the induction, we need to prove the following:

Theorem 6.1. The assertions $A_{n-1}, B_{n-1}, C_{n-1}$ and $C_{n-1}^{\circ}$ imply $A_{n}, B_{n}, C_{n}$ and $C_{n}^{\circ}$.

Proof. (i) First we prove $A_{n}$ for $k \geq 2$. By Theorem 4.7 (A)-(i), we have $f_{n, k}^{+}=$ $\lambda_{2}\left(f_{n-1, k+1}^{+}\right) \gamma_{1}\left(f_{n-1, k+1}^{-}\right)$for $k \geq 1$, where

$$
\gamma_{1}\left(f_{n-1, k+1}^{-}\right): M_{n, k}=I\left(f_{n-1, k+1}^{-}, f_{n-1, k-1}^{+}\right) \rightarrow J\left(f_{n-1, k+1}^{-}\right)
$$

and

$$
\lambda_{2}\left(f_{n-1, k+1}^{+}\right): J\left(f_{n-1, k+1}^{-}\right) \rightarrow I I\left(f_{n-1, k+1}^{+}, f_{n-1, k+1}^{-}\right)=M_{n, k+1} .
$$

By $A_{n-1},\left(f_{n-1, k-1}^{+}\right)_{*}$ is surjective for $k \geq 2$. Hence we can apply Theorem 5.6 (ii) with $s=f_{n-1, k+1}^{-}$and $t=f_{n-1, k-1}^{+}$. The first exact sequence implies that $\gamma_{1}\left(f_{n-1, k+1}^{-}\right)_{*}$ is surjective.

By $B_{n-1},\left(f_{n-1, k+1}^{-}\right)_{*}$ is injective. Hence we can apply Theorem $5.6^{\circ}$ (ii) with $p=f_{n-1, k+1}^{+}$and $q=f_{n-1, k+1}^{-}$. By the second exact sequence with $A_{n-1}$ and $B_{n-1}$, we see that $\lambda_{2}\left(f_{n-1, k+1}^{+}\right)_{*}$ is surjective for $k \geq 1$. 
Now we see that $\left(f_{n, k}^{+}\right)_{*}$ is surjective for $k \geq 2$, and hence we have shown $A_{n}$ for $k \geq 2$.

(ii) Next we show $A_{n}$ for $k=1$ and $C_{n}^{\circ}$. By $C_{n-1}$, we can apply Theorem 5.6 (ii) with $s=f_{n-1,2}^{-}$and $t=f_{n-1,0}^{+}$. The first exact sequence is

$$
\begin{aligned}
& 0 \longrightarrow \operatorname{Coker}_{j}\left(\left(f_{n-1,0}^{+}\right)_{*}\right) \oplus \operatorname{Ker}_{j-1}\left(\left(f_{n-1,0}^{+}\right)_{*}\right) \longrightarrow H_{j}\left(M_{n, 1}\right) \\
& \stackrel{\gamma_{1}\left(f_{n-1,2}^{-}\right)_{*}}{\longrightarrow} H_{j}\left(J\left(f_{n-1,2}^{-}\right)\right) \longrightarrow 0 .
\end{aligned}
$$

Since $\lambda_{2}\left(f_{n-1,2}^{+}\right)_{*}: H_{*}\left(J\left(f_{n-1, k+1}^{-}\right)\right) \rightarrow H_{*}\left(M_{n, 2}\right)$ is surjective by the argument of (i), $\left(f_{n, 1}^{+}\right)_{*}=\lambda_{2}\left(f_{n-1,2}^{+}\right)_{*} \gamma_{1}\left(f_{n-1,2}^{-}\right)_{*}$ is surjective. Thus we have shown $A_{n}$ for $k=1$.

By Theorem 4.7 (B)-(iv), we have

$$
f_{n, 1}^{-}=\gamma_{2} \gamma_{1}\left(f_{n-1,2}^{-}\right) \text {. }
$$

Since $\left(\gamma_{2}\right)_{*}: H_{*}\left(J\left(f_{n-1,2}^{-}\right)\right) \rightarrow H_{*}\left(M_{n, 0}\right)$ is injective by $B_{n-1}$, we have

$$
\operatorname{Ker}_{j}\left(\left(f_{n, 1}^{-}\right)_{*}\right) \cong \operatorname{Ker}_{j}\left(\gamma_{1}\left(f_{n-1,2}^{-}\right)_{*}\right)
$$

Now since $\left(f_{n, 1}^{+}\right)_{*}=\lambda_{2}\left(f_{n-1,2}^{+}\right)_{*} \gamma_{1}\left(f_{n-1,2}^{-}\right)_{*}$, we see that

$$
\operatorname{Ker}\left(\left(f_{n, 1}^{-}\right)_{*}\right) \subset \operatorname{Ker}\left(\left(f_{n, 1}^{+}\right)_{*}\right) \text {. }
$$

Thus we have shown $C_{n}^{\circ}$.

(iii) We can prove $B_{n}$ and $C_{n}$ similarly.

This completes the proof of Theorem 6.1.

Now we have proved the following:

Theorem 6.3. The assertions $A_{n}, B_{n}, C_{n}$ and $C_{n}^{\circ}$ hold for $n \geq 2$.

Moreover, concerning $C_{n}$ and $C_{n}^{\circ}$, we can add the following formulae. Since they are proved in the same way as in Theorem 6.1 , we omit the proof.

Theorem 6.4. (i) For an even $n$, we have

$$
\operatorname{Ker}_{j}\left(\left(f_{n, 0}^{+}\right)_{*}\right) \cong \operatorname{Ker}_{j}\left(\left(f_{n-1,1}^{+}\right)_{*}\right)
$$

(ii) For an odd $n$, we have

$$
\operatorname{Coker}_{j}\left(\left(f_{n, 1}^{-}\right)_{*}\right) \cong \operatorname{Coker}_{j-1}\left(\left(f_{n-1,2}^{-}\right)_{*}\right) .
$$

Theorem 6.3 tells us that we can apply Theorems 5.6 (i) and $5.6^{\circ}$ (i) to $H_{*}\left(M_{n, k}\right)$. Then by using Theorems 6.3 and 6.4 , we can write down $H_{*}\left(M_{n, k}\right)$ as follows.

Theorem 6.5. (i) For $n-k \equiv 0 \bmod 2$ and $k \geq 2$,

$$
H_{j}\left(M_{n, k}\right) \cong H_{j}\left(M_{n-1, k}\right) \oplus H_{j-1}\left(M_{n-1, k+1}\right) \oplus \operatorname{Ker}_{j-1}\left(\left(f_{n-1, k-1}^{+}\right)_{*}\right),
$$

where $\left(f_{n-1, k-1}^{+}\right)_{*}: H_{*}\left(M_{n-1, k-1}\right) \rightarrow H_{*}\left(M_{n-1, k}\right)$ is surjective.

(ii) For $n-k \equiv 1 \bmod 2$ and $k \geq 2$,

$$
H_{j}\left(M_{n, k}\right) \cong H_{j}\left(M_{n-1, k+1}\right) \oplus \operatorname{Coker}_{j}\left(\left(f_{n-1, k}^{-}\right)_{*}\right) \oplus H_{j-1}\left(M_{n-1, k}\right),
$$

where $\left(f_{n-1, k}^{-}\right)_{*}: H_{*}\left(M_{n-1, k}\right) \rightarrow H_{*}\left(M_{n-1, k-1}\right)$ is injective. 
(iii)

$$
\begin{aligned}
H_{j}\left(M_{2 m+1,1}\right) \cong & H_{j}\left(M_{2 m, 1}\right) \oplus \operatorname{Coker}_{j}\left(\left(f_{2 m, 0}^{+}\right)_{*}\right) \\
& \oplus H_{j-1}\left(M_{2 m, 2}\right) \oplus \operatorname{Ker}_{j-1}\left(\left(f_{2 m, 0}^{+}\right)_{*}\right),
\end{aligned}
$$

where

$$
\operatorname{Ker}_{j-1}\left(\left(f_{2 m, 0}^{+}\right)_{*}\right) \cong \operatorname{Ker}_{j-1}\left(\left(f_{2 m-1,1}^{+}\right)_{*}\right)
$$

and $\left(f_{2 m-1,1}^{+}\right)_{*}: H_{*}\left(M_{2 m-1,1}\right) \rightarrow H_{*}\left(M_{2 m-1,2}\right)$ is surjective.

$$
\begin{aligned}
H_{j}\left(M_{2 m, 1}\right) \cong & H_{j}\left(M_{2 m-1,2}\right) \oplus \operatorname{Coker}_{j}\left(\left(f_{2 m-1,1}^{-}\right)_{*}\right) \oplus H_{j-1}\left(M_{2 m-1,1}\right) \\
& \oplus \operatorname{Ker}_{j-1}\left(\left(f_{2 m-1,1}^{-}\right)_{*}\right)
\end{aligned}
$$

where

$$
\operatorname{Coker}_{j}\left(\left(f_{2 m-1,1}^{-}\right)_{*}\right) \cong \operatorname{Coker}_{j-1}\left(\left(f_{2 m-2,2}^{-}\right)_{*}\right)
$$

and $\left(f_{2 m-2,2}^{-}\right)_{*}: H_{*}\left(M_{2 m-2,2}\right) \rightarrow H_{*}\left(M_{2 m-2,1}\right)$ is injective.

Now we give recurrence relations for $H_{*}\left(M_{n, k} ; K\right)$. When we have fixed a field $K$, we denote the Poincaré polynomial of $M_{n, k}$ with $K$-coefficients by $P S(n, k)$. Thus $P S(n, k)=\sum_{\lambda} \operatorname{dim}_{K} H_{\lambda}\left(M_{n, k} ; K\right) t^{\lambda}$. We note the following initial conditions.

(i) $P S(1, k)=0$ for $k \in \mathbf{N} \cup\{0\}$.

(ii) $P S(2,1)=1$, while $P S(2, k)=0$ for $k \in \mathbf{N} \cup\{0\}$ and $k \neq 1$.

Then our recurrence relations are given by the following:

Theorem 6.7. (I) For $m \geq 1$, we have

$$
\begin{aligned}
P S(2 m+1,0) & =(1+t) P S(2 m, 1), \\
P S(2 m+1,1) & =-(1+t) P S(2 m-1,2)+2 P S(2 m, 1)+t P S(2 m, 2), \\
P S(2 m+1,2 i+1) & =t P S(2 m, 2 i)+(1-t) P S(2 m, 2 i+1)+t P S(2 m, 2 i+2), \\
P S(2 m+1,2 i) & =P S(2 m, 2 i-1)+(t-1) P S(2 m, 2 i)+P S(2 m, 2 i+1), \\
P S(2 m+1,2 m) & =1 .
\end{aligned}
$$

(II) For $m \geq 2$, we have

$$
\begin{aligned}
P S(2 m, 0)= & (1+t) P S(2 m-1,1), \\
P S(2 m, 1)= & P S(2 m-1,2)+t P S(2 m-1,1)+(1+t) t(P S(2 m-2,1) \\
& -P S(2 m-2,2))+t(P S(2 m-1,1)-P S(2 m-1,0)), \\
P S(2 m, 2 i+1)= & P S(2 m-1,2 i)+(t-1) P S(2 m-1,2 i+1) \\
& +P S(2 m-1,2 i+2), \\
P S(2 m, 2 i)= & +P S(2 m-1,2 i-1)+(1-t) P S(2 m-1,2 i) \\
& +t P S(2 m-1,2 i+1), \\
P S(2 m, 2 m-1)= & 1 .
\end{aligned}
$$

Proof. We prove only $P S(2 m, 1)$. From Theorem 6.5 (iv), we have

$$
\begin{aligned}
P S(2 m, 1)= & P S(2 m-1,2)+P S\left(\operatorname{Coker}\left(\left(f_{2 m-1,1}^{-}\right)_{*}\right)\right)+t P S(2 m-1,1) \\
& +t P S\left(\operatorname{Ker}\left(\left(f_{2 m-1,1}^{-}\right)_{*}\right)\right)
\end{aligned}
$$


and

$$
P S\left(\text { Coker }\left(\left(f_{2 m-1,1}^{-}\right)_{*}\right)\right)=t(P S(2 m-2,1)-P S(2 m-2,2)) .
$$

Note that we have

$$
\begin{aligned}
\left.\operatorname{dim} \operatorname{Ker}_{j}\left(f_{2 m-1,1}^{-}\right)_{*}\right)= & \left.\operatorname{dim} \operatorname{Coker}_{j}\left(f_{2 m-1,1}^{-}\right)_{*}\right)+\operatorname{dim} H_{j}\left(M_{2 m-1,1}\right) \\
& -\operatorname{dim} H_{j}\left(M_{2 m-1,0}\right)
\end{aligned}
$$

for $\left(f_{2 m-1,1}^{-}\right)_{*}: H_{j}\left(M_{2 m-1,1}\right) \rightarrow H_{j}\left(M_{2 m-1,0}\right)$. Hence we have

$$
\begin{aligned}
P S\left(\operatorname{Ker}\left(\left(f_{2 m-1,1}^{-}\right)_{*}\right)\right)= & t(P S(2 m-2,1)-P S(2 m-2,2))+P S(2 m-1,1) \\
& -P S(2 m-1,0) .
\end{aligned}
$$

Now the result follows easily.

Now the solution of the recurrence relations of Theorem 6.7 under the initial conditions (6.6) is given by the following:

Theorem 6.8. (I) For $m \geq 0$, we have

$$
\begin{aligned}
P S(2 m+1,0)= & \sum_{\lambda=0}^{m-2}\left(\begin{array}{c}
2 m \\
\lambda
\end{array}\right) t^{\lambda}+\left\{\left(\begin{array}{c}
2 m \\
m-1
\end{array}\right)+\left(\begin{array}{c}
2 m-1 \\
m-2
\end{array}\right)\right\} t^{m-1} \\
& +\left\{\left(\begin{array}{c}
2 m \\
m-1
\end{array}\right)+\left(\begin{array}{c}
2 m-1 \\
m-3
\end{array}\right)\right\} t^{m}+\sum_{\lambda=m+1}^{2 m-2}\left(\begin{array}{c}
2 m \\
\lambda+2
\end{array}\right) t^{\lambda}, \\
P S(2 m+1,1)= & \sum_{\lambda=0}^{m-2}\left(\begin{array}{c}
2 m \\
\lambda
\end{array}\right) t^{\lambda}+2\left(\begin{array}{c}
2 m \\
m-1
\end{array}\right) t^{m-1}+\sum_{\lambda=m}^{2 m-2}\left(\begin{array}{c}
2 m \\
\lambda+2
\end{array}\right) t^{\lambda}, \\
P S(2 m+1,2 i+1)= & \sum_{\lambda=0}^{m-i-1}\left(\begin{array}{c}
2 m \\
\lambda
\end{array}\right) t^{\lambda}+\sum_{\lambda=m+i-1}^{2 m-2}\left(\begin{array}{c}
2 m \\
\lambda+2
\end{array}\right) t^{\lambda}(1 \leq i \leq m-1), \\
P S(2 m+1,2 i)= & \sum_{\lambda=0}^{m-i}\left(\begin{array}{c}
2 m \\
\lambda
\end{array}\right) t^{\lambda}+\sum_{\lambda=m+i-1}^{2 m-2}\left(\begin{array}{c}
2 m \\
\lambda+2
\end{array}\right) t^{\lambda}(1 \leq i \leq m) .
\end{aligned}
$$

(II) For $m \geq 1$, we have

$$
\begin{aligned}
P S(2 m, 0)= & \sum_{\lambda=0}^{m-3}\left(\begin{array}{c}
2 m-1 \\
\lambda
\end{array}\right) t^{\lambda}+\left\{2\left(\begin{array}{c}
2 m-2 \\
m-2
\end{array}\right)+\left(\begin{array}{c}
2 m-3 \\
m-4
\end{array}\right)\right. \\
& \left.+\left(\begin{array}{c}
2 m-3 \\
m-3
\end{array}\right)\right\}\left(t^{m-2}+t^{m-1}\right)+\sum_{\lambda=m}^{2 m-3}\left(\begin{array}{c}
2 m-1 \\
\lambda+2
\end{array}\right) t^{\lambda}, \\
P S(2 m, 1)= & \sum_{\lambda=0}^{m-2}\left(\begin{array}{c}
2 m-1 \\
\lambda
\end{array}\right) t^{\lambda}+\left(\begin{array}{c}
2 m \\
m-1
\end{array}\right) t^{m-1}+\sum_{\lambda=m}^{2 m-3}\left(\begin{array}{c}
2 m-1 \\
\lambda+2
\end{array}\right) t^{\lambda}, \\
P S(2 m, 2 i+1)= & \sum_{\lambda=0}^{m-i-1}\left(\begin{array}{c}
2 m-1 \\
\lambda
\end{array}\right) t^{\lambda}+\sum_{\lambda=m+i-1}^{2 m-3}\left(\begin{array}{c}
2 m-1 \\
\lambda+2
\end{array}\right) t^{\lambda} \quad(1 \leq i \leq m-1), \\
P S(2 m, 2 i)= & \sum_{\lambda=0}^{m-i-1}\left(\begin{array}{c}
2 m-1 \\
\lambda
\end{array}\right) t^{\lambda}+\sum_{\lambda=m+i-2}^{2 m-3}\left(\begin{array}{c}
2 m-1 \\
\lambda+2
\end{array}\right) t^{\lambda} \quad(1 \leq i \leq m-1) .
\end{aligned}
$$


Proof. We can prove Theorem 6.8 easily by induction on $n$ (= the number of vertices, i.e., $n=2 m$ or $2 m+1$ ).

Theorem 6.8 shows that $P S(n, k)$ does not depend on the coefficient field $K$. Hence we see that $H_{*}\left(M_{n, k} ; \mathbf{Z}\right)$ is a torsion free module, and Theorem B follows.

Again by Theorem 6.8 , we have determined $H_{*}\left(M_{n, k} ; \mathbf{Z}\right)$. In particular, we have Theorem C.

\section{ACKNOWLEDGMENTS}

The first and second authors are grateful to the Mathematics Departments of the University of Rochester and Stanford University for their hospitality while this work was being carried out. The authors would also like to thank the referee for giving detailed suggestions on how to improve the original. In particular, $\S \S 4$ and 5 in the first draft have been completely revised according to his or her arguments.

\section{REFERENCES}

[1] A. Bousfield and D. Kan, Homotopy limits, completions and localizations, Lecture Notes in Math., vol. 304, Springer-Verlag, 1972. MR 51:1825

[2] J.-C. Hausmann, Sur la topologie des bras articulés, Algebraic Topology, Poznań 1989, Lecture Notes in Math., vol. 1474, Springer-Verlag, 1991, pp. 146-159. MR 93a:57035

[3] T. Havel, Some examples of the use of distances as coordinates in Euclidean geometry, Journal of Symbolic Computation 11 (1991), 579-593. MR 92j:51033

[4] Y. Kamiyama, An elementary proof of a theorem of T. F. Havel, Ryukyu Math. J. 5 (1992), 7-12. MR 94a:52044

[5] Y. Kamiyama, Topology of equilateral polygon linkages, Top. and its Applications 68 (1996), 13-31. MR 96j:52041

[6] M. Kapovich and J. Millson, On the moduli space of polygons in the Euclidean plane, Journal of Diff. Geometry 42 (1995), 133-164. MR 98b:52019

[7] M. Kato, Topology of k-regular spaces and algebraic sets, Manifolds-Tokyo 1973, Univ. of Tokyo Press, Tokyo, 1975, pp. 153-159. MR 54:6149

[8] J. Milnor, Morse theory, Ann. of Math. Studies, vol. 51, Princeton Univ. Press, Princeton, 1963. MR 29:634

[9] J. Milnor, Singular points of complex hypersurfaces, Ann. of Math. Studies, vol. 61, Princeton Univ. Press, Princeton, 1968. MR 39:969

[10] I. Schoenberg, Linkages and distance geometry. I. Linkages, Indag. Math. 31 (1969), 42-52. MR 39:7512

[11] T. Toma, An analogue of a theorem of T. F. Havel, Ryukyu Math. J. 6 (1993), 69-77; Corrections, ibid. 8 (1995), 95-96. MR 95g:52038

Department of Mathematics, University of the Ryukyus, Nishihara-Cho, Okinawa 903-01, JAPAN

E-mail address: kamiyama@sci.u-ryukyu.ac.jp

Department of Mathematics, University of the Ryukyus, Nishinara-Cho, Okinawa 903-01, JAPAN

E-mail address: tez@sci.u-ryukyu.ac.jp 